\title{
An inverse anisotropic conductivity problem induced by twisting a homogeneous cylindrical domain
}

\author{
Mourad Choulli and Eric Soccorsi
}

\begin{abstract}
We consider the inverse problem of determining the unknown function $\alpha: \mathbb{R} \rightarrow \mathbb{R}$ from the DN map associated with the operator $\operatorname{div}\left(A\left(x^{\prime}, \alpha\left(x_{3}\right)\right) \nabla \cdot\right)$ acting in the infinite straight cylindrical waveguide $\Omega=\omega \times \mathbb{R}$, where $\omega$ is a bounded domain of $\mathbb{R}^{2}$. Here $A=\left(A_{i j}(x)\right), x=\left(x^{\prime}, x_{3}\right) \in \Omega$, is a matrix-valued metric on $\Omega$ obtained by straightening a twisted waveguide. This inverse anisotropic conductivity problem remains generally open, unless the unknown function $\alpha$ is assumed to be constant. In this case we prove Lipschitz stability in the determination of $\alpha$ from the corresponding DN map. The same result remains valid upon substituting a suitable approximation of the DN map, provided the function $\alpha$ is sufficiently close to some a priori fixed constant.
\end{abstract}

Mathematics Subject Classification (2010). 35R30.

Keywords. Dirichlet Laplacian, twisted infinite cylindrical domain, inverse anisotropic conductivity problem, DN map, stability estimate.

\section{Contents}

1 Introduction . . . . . . . . . . . . . . . . 296

2 The DN map $\Lambda_{\alpha}$. . . . . . . . . . . . . . . . . . . . . . 299

3 Can we determine the unknown function $\alpha$ from $\Lambda_{\alpha}$ ? . . . . . . . 303

4 The case of constant unknown functions . . . . . . . . . . . . . . . 309

A Restriction to $\tilde{H}^{3 / 2}(\partial \Omega) \ldots \ldots$. . . . . . . . . . . . . 320

B Linking $\Lambda_{\alpha}$ to the DN map associated with $\Omega_{\theta} \ldots \ldots$. . . . . . . . 324

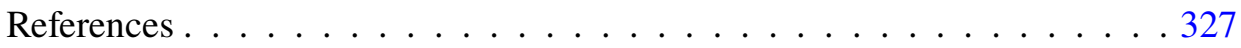




\section{Introduction}

In the present paper we consider an inverse conductivity problem in an anisotropic medium arising from the twisting of a homogeneous infinite straight cylindrical waveguide. Generically the inverse conductivity problem in an arbitrary domain $\Omega \subset \mathbb{R}^{n}, n \geq 1$, is to determine a symmetric, positive definite matrix $A=A(x)$, $x \in \Omega$, representing the conductivity tensor of $\Omega$, from the Dirichlet-to-Neumann (abbreviated to DN in the following) map associated with $A$ :

$$
\Lambda_{A}: u_{\mid \partial \Omega} \longmapsto A \nabla u \cdot v .
$$

Here $u$ is the solution to the elliptic equation $\operatorname{div}(A \nabla u)=0$ in $\Omega$ and $v$ denotes the unit outward normal vector to the boundary $\partial \Omega$ of $\Omega$.

If $A$ is isotropic (i.e. $A$ is the identity matrix of $\mathbb{R}^{n}$ up to some multiplicative scalar -unknown- function of $x \in \Omega$ ), Sylvester and Uhlmann [36] proved that the conductivity is uniquely determined from the knowledge of $\Lambda_{A}$. Similar results were derived by Lionheart in [28] upon substituting a suitable given matrix $A_{0}(x)$ for the identity. For a conductivity of the form $A(x)=A_{0}(x, \alpha(x))$ where $A_{0}$ is given and $\alpha$ is an unknown scalar function, Alessandrini and Gaburro [2,3] obtained uniqueness and stability under the monotonicity assumption

$$
\partial_{t} A_{0}(x, t) \geq c I, \quad x \in \Omega, t \in \mathbb{R} .
$$

Here $c$ is some positive constant and $I$ stands for the identity of $\mathbb{R}^{n}$. The case $A(x)=A_{0}(\alpha(x))$ was treated earlier by Alessandrini in [1] under the same kind of monotonicity condition.

All the above mentioned results were obtained in a bounded domain of $\mathbb{R}^{n}$. In this paper we will rather consider an infinite cylindrical straight waveguide $\Omega=\omega \times \mathbb{R}$, where $\omega$ is a bounded domain of $\mathbb{R}^{2}$. To the variable twisting angle $\theta \in C^{1}(\mathbb{R})$ we then associate the twisted waveguide

$$
\Omega_{\theta}=\left\{\left(R_{\theta\left(x_{3}\right)} x^{\prime}, x_{3}\right) ; x^{\prime}=\left(x_{1}, x_{2}\right) \in \omega, x_{3} \in \mathbb{R}\right\},
$$

where $R_{\xi}$ denotes the rotation in $\mathbb{R}^{2}$ of angle $\xi \in \mathbb{R}$. Twisted waveguides modeled by $\Omega_{\theta}$ exhibit interesting propagation properties such as the occurence of propagating modes with phase velocities slower than those of similar modes in a straight waveguide. This explains why these peculiar structures are at the center of the attention of many theoretical and applied physicists (see e.g. [8, 20, 24, 31, $33,37,38]$ ). From a mathematical viewpoint twisted waveguides are the source of challenging spectral and PDE problems, some of them having been extensively 
studied in the mathematical literature (see e.g. [6, 11, 21, 22, 23, 25]). Moreover the cylindrical geometry is well suited to the construction of complex geometrical optics solutions $[9,7]$, which motivates for the analysis of inverse problems occurring in waveguides.

We consider the following boundary value problem (abbreviated to BVP) for the Laplacian in $\Omega_{\theta}$ :

$$
\begin{cases}\Delta v(y)=0, & y \in \Omega_{\theta}, \\ v(y)=g(y), & y \in \partial \Omega_{\theta} .\end{cases}
$$

Upon straightening $\Omega_{\theta}$, (1.2) may be brought into an equivalent BVP stated in $\Omega$. This can be seen by introducing

$$
T_{\xi}=\left(\begin{array}{cc}
R_{\xi} & 0 \\
0 & 1
\end{array}\right)
$$

putting $u(x)=v\left(T_{\theta\left(x_{3}\right)}\left(x^{\prime}, x_{3}\right)\right), x=\left(x^{\prime}, x_{3}\right) \in \Omega$, and performing the change of variable $y=T_{\theta\left(x_{3}\right)}\left(x^{\prime}, x_{3}\right)$. We get using direct calculation that $u$ is solution to the following elliptic BVP in the divergence form

$$
\begin{cases}\operatorname{div}\left(A_{0}\left(x^{\prime}, \alpha\left(x_{3}\right)\right) \nabla u\right)=0, & x \in \Omega, \\ u(x)=f(x), & x \in \partial \Omega,\end{cases}
$$

where $\alpha=\theta^{\prime}, f(x)=g\left(T_{\theta\left(x_{3}\right)}\left(x^{\prime}, x_{3}\right)\right)$ for $x \in \partial \Omega$, and the matrix $A_{0}$ is given by

$$
A_{0}\left(x^{\prime}, t\right)=\left(\begin{array}{ccc}
1+x_{2}^{2} t^{2} & -x_{2} x_{1} t^{2} & -x_{2} t \\
-x_{2} x_{1} t^{2} & 1+x_{1}^{2} t^{2} & x_{1} t \\
-x_{2} t & x_{1} t & 1
\end{array}\right), \quad x^{\prime} \in \omega, t \in \mathbb{R} .
$$

At this point it is worth stressing out that the BVP (1.3) is stated on the straight waveguide $\Omega$ and not on the twisted waveguide $\Omega_{\theta}$ itself. Nevertheless we note that the geometry of $\Omega_{\theta}$ is expressed in (1.3) through the metric $A=\left(A_{i j}(x)\right)=$ $A_{0}\left(x^{\prime}, \alpha\left(x_{3}\right)\right)$.

The problem we examine in this paper is to know whether the unknown function $\alpha$ can be determined from the DN map

$$
\Lambda_{\alpha}: f \longmapsto A \nabla u \cdot v .
$$

This is the same kind of inverse anisotropic conductivity problem, but stated here in the unbounded straight waveguide $\Omega$, as the one studied in $[1,2,3,12]$ in a bounded domain. However, it turns out that the usual monotonicity assumption (1.1) on the conductivity, which is essential to the identification of $A$ from 
the DN map in this approach, is not verified in our framework (since the matrix $\partial_{t} A_{0}\left(x^{\prime}, t\right)$ has a negative eigenvalue $\left|x^{\prime}\right|^{2} t-\sqrt{\left|x^{\prime}\right|^{4} t^{2}+\left|x^{\prime}\right|^{2}}$, see (3.15)). This explains why the inverse problem associated with (1.3) is still open for general unknown functions $\alpha \in C(\mathbb{R})$. However, if $\alpha$ is sufficiently close to some a priori fixed constant we prove in Theorem 3.1 that this unknown function may well be determined from the knowledge of some suitable approximation of the DN map; see (3.17). Moreover, in the particular case where $\alpha$ is known to be constant so the conductivity matrix $A$ is invariant with respect to the infinite variable $x_{3}$, the original problem is equivalent to some inverse anisotropic conductivity problem stated in $\omega$. The corresponding conductivity matrix satisfies a weak monotonicity condition in this case implying Lipschitz stability in the determination of $\alpha$ from $\Lambda_{\alpha}$ (see Theorem 4.1).

The problem under investigation in this paper is a special case of the anisotropic Calderón problem, which consists in determining the geometric structure of a smooth Riemannian manifold with boundary from the Cauchy data of harmonic functions. Actually, since the DN mapping is invariant under diffeomorphisms which preserve the boundary, it is well known that there is an obstruction to uniqueness in this problem. Nevertheless, in dimensions greater than or equal to three, it was proved by Lee and Uhlmann [27] that compact real-analytic manifolds are uniquely defined by the DN map up to diffeomorphisms preserving the boundary. A similar result was obtained in [15] by Guillarmou and Sà Barreto for Einstein metrics that are real-analytic in the interior. The case of non-analytic simple manifolds was treated in [9] by means of Carleman estimates with limiting weights. Recently, Dos Santos Ferreira, Kurylev, Lassas and Salo proved in [10] that the boundary measurements in an infinite cylinder uniquely determine the transversal metric. We point out that in dimension two, there is an additional obstruction to uniqueness for the anisotropic Calderón problem, arising from the conformal invariance of the associated Laplace-Beltrami operator. See $[34,35,26]$ and references therein for a detailed study of this case, and [4] for a specific treatment of the same inverse problem in the plane. Finally, we refer to $[18,19]$ for inverse anisotropic conductivity problems with partial Cauchy data.

The paper is organized as follows. Section 2 gathers the definition and the main properties of $\Lambda_{\alpha}$. In the first part of Section 3 we adapt the method developed by Alessandrini and Gaburro in $[2,3]$ to determine the conductivity in a bounded anisotropic medium from the DN map, to the particular framework of an infinite cylindrical waveguide under consideration in this text. Although this technique does not allow for the identification of general unknown functions $\alpha$, we prove stability in the determination of $\alpha$ from some suitable approximation of the DN 
map, provided $\alpha$ is sufficiently close to some arbitrarily fixed constant. The case of constant unknown functions is examined in Section 4 and we prove Lipschitz stability in the determination of $\alpha$ from the knowledge of $\Lambda_{\alpha}$ in this case. Finally, for the sake of completeness, we gather several properties (which are not directly useful for the analysis of the inverse problem under study) of the DN map $\Lambda_{\alpha}$ in Appendix.

Acknowledgments. We are strongly indebted to the unknown referee of this paper for numerous remarks which have been helpful to us in improving this text. We also want to thank Günther Uhlmann for valuable suggestions and comments during the completion of this work.

\section{The DN map $\Lambda_{\alpha}$}

Solution to the BVP (1.3). As we are dealing with an infinitely extended domain $\Omega$, we start by defining the Sobolev spaces on $\partial \Omega$ required by our analysis. Let $s$ be either $1 / 2$ or $3 / 2$. Since the trace operator

$$
\begin{aligned}
\tau: C_{0}^{\infty}\left(\mathbb{R}, H^{s+1 / 2}(\omega)\right) & \longrightarrow L^{2}\left(\mathbb{R}, H^{s}(\partial \omega)\right), \\
G & \longmapsto\left[t \in \mathbb{R} \mapsto G(t, \cdot)_{\mid \partial \omega}\right],
\end{aligned}
$$

extends to a bounded operator, still denoted by $\tau$, from $L^{2}\left(\mathbb{R}, H^{s+1 / 2}(\omega)\right)$ into $L^{2}\left(\mathbb{R}, H^{s}(\partial \omega)\right)$, we put

$$
\begin{aligned}
\widetilde{H}^{s}(\partial \Omega)= & \left\{g \in L^{2}\left(\mathbb{R}, H^{s}(\partial \omega)\right) ; \text { there exists } G \in H^{s+1 / 2}(\Omega)\right. \\
& \text { such that } \tau G=g\} .
\end{aligned}
$$

The space $\widetilde{H}^{s}(\partial \Omega)$ is Hilbertian (e.g. [32, p. 398]) for the quotient norm

$$
\|g\|_{\widetilde{H}^{s}(\partial \Omega)}=\min \left\{\|G\|_{H^{s+1 / 2}(\Omega)} ; G \in H^{s+1 / 2}(\Omega) \text { is such that } \tau G=g\right\} .
$$

For the sake of simplicity we write $G=g$ on $\partial \Omega$ instead of $\tau G=g$ in the sequel.

Remark 2.1. The definition in [29, Chap. 1] of fractional Sobolev spaces $H^{s}(\partial \Omega)$, $s \in \mathbb{Q}$, on compact manifolds without boundary, may as well be adapted to the manifold $\partial \Omega=\partial \omega \times \mathbb{R}$ and it is not hard to check that the two spaces $H^{s}(\partial \Omega)$ and $\widetilde{H}^{s}(\partial \Omega)$ then coincide both algebraically and topologically in this case. 
Remark 2.2. There is a minimizer to equation (2.2) for any arbitrary $g \in \widetilde{H}^{s}(\partial \Omega)$ with $s \in\{1 / 2,3 / 2\}$. Indeed, the mapping $\tau$ is continuous from $H^{s+1 / 2}(\Omega)$ into $L^{2}\left(\mathbb{R}, H^{s+1 / 2}(\omega)\right)$ so its kernel is closed in $H^{s+1 / 2}(\Omega)$. Then $\left\{G \in H^{s+1 / 2}(\Omega)\right.$, $\tau G=g\}$ is a closed affine subset of $H^{s+1 / 2}(\Omega)$. This yields the existence of a minimizer to (2.2) since there is always an element of minimal norm in a closed convex subset of an Hilbert space.

As a direct consequence of equations (2.1) and (2.2) and Remark 2.2, we have the following extension lemma.

Lemma 2.1. Let $g \in \widetilde{H}^{s}(\partial \Omega)$ for $s \in\{1 / 2,3 / 2\}$. Then there exists $G \in H^{s+1 / 2}(\Omega)$ such that $G=g$ on $\partial \Omega$ and

$$
\|G\|_{H^{s+1 / 2(\Omega)}}=\|g\|_{\widetilde{H}^{s}(\partial \Omega)} .
$$

The main ingredient in the analysis of the BVP (1.3) is the uniform ellipticity of $A$, where $A$ denotes either $A\left(x^{\prime}, t\right)$ or $A_{0}\left(x^{\prime}, \alpha\left(x_{3}\right)\right)$, as defined in Section 1. Indeed, for all $\zeta \in \mathbb{R}^{3}$ we have

$$
\begin{aligned}
A\left(x^{\prime}, t\right) \zeta \cdot \zeta & =\zeta_{1}^{2}+\zeta_{2}^{2}+\zeta_{3}^{2}-2 t x_{2} \zeta_{1} \zeta_{3}+2 t x_{1} \zeta_{2} \zeta_{3}+t^{2}\left(x_{2} \zeta_{1}-x_{1} \zeta_{2}\right)^{2} \\
& =\zeta_{1}^{2}+\zeta_{2}^{2}+\left(\zeta_{3}+t\left(x_{2} \zeta_{1}-x_{1} \zeta_{2}\right)\right)^{2}, \quad x^{\prime}=\left(x_{1}, x_{2}\right) \in \omega, t \in \mathbb{R},
\end{aligned}
$$

by straightforward computations, which entails that $A\left(x^{\prime}, t\right) \zeta \cdot \zeta=0$ if and only if $\zeta=0$. Since $\bar{\omega} \times[\underline{t}, \bar{t}]$ is compact for all real numbers $\underline{t}<\bar{t}$, there is thus $\lambda \geq 1$, depending on $\omega, \underline{t}$ and $\bar{t}$, such that we have

$$
\lambda^{-1}|\zeta|^{2} \leq A\left(x^{\prime}, t\right) \zeta \cdot \zeta \leq \lambda|\zeta|^{2}, \quad \text { for all } x^{\prime} \in \omega, t \in[\underline{t}, \bar{t}], \zeta \in \mathbb{R}^{3} .
$$

We turn now to studying the direct problem associated with (1.3). We pick $f \in$ $\tilde{H}^{1 / 2}(\partial \Omega)$ and $F \in H^{1}(\Omega)$ such that $F=f$ on $\partial \Omega$. In light of (2.3) and the Lax-Milgram lemma, there exists a unique $v \in H_{0}^{1}(\Omega)$ solving the variational problem

$$
\int_{\Omega} A \nabla v \cdot \nabla w d x=-\int_{\Omega} A \nabla F \cdot \nabla w d x, \quad \text { for all } w \in H_{0}^{1}(\Omega) .
$$

Hence $u=v+F$ is the unique weak solution to the BVP (1.3). That is, $u$ satisfies the first equation in (1.3) in the distributional sense and the second equation in the trace sense. By taking $w=v$ in (2.4), we get $\|v\|_{H^{1}(\Omega)} \leq C\|F\|_{H^{1}(\Omega)}$ from (2.3) and Poincaré's inequality ${ }^{1}$, whence

$$
\|u\|_{H^{1}(\Omega)} \leq C\|F\|_{H^{1}(\Omega)},
$$

${ }^{1}$ Which holds true for $\Omega$ since $\omega$ is bounded. 
where $C$ denotes some generic positive constant depending only on $\omega$. Finally, by choosing $F \in H^{1}(\Omega)$ in accordance with Lemma 2.1 so that

$$
\|F\|_{H^{1}(\Omega)}=\|f\|_{\tilde{H}^{1 / 2}(\partial \Omega)},
$$

we find out that

$$
\|u\|_{H^{1}(\Omega)} \leq C\|f\|_{\widetilde{H}^{1 / 2}(\partial \Omega)} .
$$

Definition of the DN map. Let us first introduce the following $H$ (div)-type space,

$$
H\left(\operatorname{div}_{A}, \Omega\right)=\left\{P \in L^{2}(\Omega)^{3} ; \operatorname{div}(A P) \in L^{2}(\Omega)\right\},
$$

and prove that $P \in C_{0}^{\infty}(\bar{\Omega}) \mapsto A P \cdot v \in C^{\infty}(\partial \Omega)$ may be extended to a bounded operator from $H\left(\operatorname{div}_{A}, \Omega\right)$ into the space $\widetilde{H}^{-1 / 2}(\partial \Omega)$ dual of $\widetilde{H}^{1 / 2}(\partial \Omega)$.

Proposition 2.1. Let $P \in H\left(\operatorname{div}_{A}, \Omega\right)$. Then $A P \cdot v \in \widetilde{H}^{-1 / 2}(\partial \Omega)$ and we have

$$
\|A P \cdot v\|_{\tilde{H}^{-1 / 2}(\partial \Omega)} \leq C\left(\|P\|_{L^{2}(\Omega)}+\|\operatorname{div}(A P)\|_{L^{2}(\Omega)}\right),
$$

for some constant $C=C(\omega, \theta)>0$. In addition, the identity

$$
\langle A P \cdot v, g\rangle=\int_{\Omega} G \operatorname{div}(A P) d x+\int_{\Omega} A \nabla G \cdot P d x,
$$

holds for $g \in \widetilde{H}^{1 / 2}(\partial \Omega)$ and $G \in H^{1}(\Omega)$ such that $G=g$ on $\partial \Omega$. Here $\langle\cdot, \cdot\rangle$ denotes the duality pairing between $\widetilde{H}^{1 / 2}(\partial \Omega)$ and its dual $\widetilde{H}^{-1 / 2}(\partial \Omega)$.

Proof. We first consider the case of $P \in C_{0}^{\infty}(\bar{\Omega})^{3}$. Fix $g \in \widetilde{H}^{1 / 2}(\partial \Omega)$ and choose $G \in H^{1}(\Omega)$ such that $G=g$ on $\partial \Omega$. Since $P$ has a compact support, we have

$$
\int_{\Omega} G \operatorname{div}(A P) d x=-\int_{\Omega} A \nabla G \cdot P d x+\int_{\partial \Omega} g A P \cdot v d \sigma,
$$

by Green's formula, whence

$$
\left|\int_{\partial \Omega} g A P \cdot v d \sigma\right| \leq C\|G\|_{H^{1}(\Omega)}\left(\|P\|_{L^{2}(\Omega)}+\|\operatorname{div}(A P)\|_{L^{2}(\Omega)}\right) .
$$

Taking the infimum over $\left\{G \in H^{1}(\Omega), G=g\right.$ on $\left.\partial \Omega\right\}$ in the right hand side of above estimate, we find that (2.6) holds true for every $P \in C_{0}^{\infty}(\bar{\Omega})^{3}$.

Further, we pick $P \in H\left(\operatorname{div}_{A}, \Omega\right)$. The set $C_{0}^{\infty}(\bar{\Omega})^{3}$ being dense in $H\left(\operatorname{div}_{A}, \Omega\right)$, as can be seen by mimicking the proof of [13, Theorem 2.4], we may find a sequence $\left(P_{k}\right)_{k}$ in $C_{0}^{\infty}(\bar{\Omega})^{3}$ converging to $P$ in $H\left(\operatorname{div}_{A}, \Omega\right)$. Moreover, $\left(A P_{k} \cdot v\right)_{k}$ being a Cauchy sequence in $\widetilde{H}^{-1 / 2}(\partial \Omega)$ by (2.6), then $\left(A P_{k} \cdot v\right)_{k}$ admits a limit, denoted by $A P \cdot \nu$, in $\widetilde{H}^{-1 / 2}(\partial \Omega)$. Now (2.7) follows readily from this and (2.8). 
Let $u$ be the $H^{1}(\Omega)$-solution to (1.3). Applying Proposition 2.1 to $P=\nabla u$, we deduce from (2.5) that

$$
\Lambda_{\alpha}: f \longmapsto A \nabla u \cdot v
$$

is well defined as a bounded operator from $\widetilde{H}^{1 / 2}(\partial \Omega)$ into $\widetilde{H}^{-1 / 2}(\partial \Omega)$. Moreover the following identity

$$
\left\langle\Lambda_{\alpha} f, g\right\rangle=\int_{\Omega} A \nabla u \cdot \nabla G d x,
$$

holds for all $g \in \widetilde{H}^{1 / 2}(\partial \Omega)$ and $G \in H^{1}(\Omega)$ such that $G=g$ on $\partial \Omega$.

Further, by taking $G=v$ in (2.9), where $v$ is the solution to (1.3) with $f$ replaced by $g$, we find out that

$$
\left\langle\Lambda_{\alpha} f, g\right\rangle=\int_{\Omega} A \nabla u \cdot \nabla v d x=\int_{\Omega} \nabla u \cdot A \nabla v d x,
$$

entailing

$$
\left\langle\Lambda_{\alpha} f, g\right\rangle=\left\langle f, \Lambda_{\alpha} g\right\rangle, \quad \text { for all } f, g \in \widetilde{H}^{1 / 2}(\partial \Omega) .
$$

This proves that $\left.\Lambda_{\alpha}^{*}\right|_{\tilde{H}^{1 / 2}(\partial \Omega)}=\Lambda_{\alpha}$, where $\widetilde{H}^{1 / 2}(\partial \Omega)$ is identified with a subspace of its bidual space.

Finally, for $i=1,2$, we put

$$
A_{i}=A\left(x^{\prime}, \alpha_{i}\left(x_{3}\right)\right) \quad \text { and } \quad \Lambda_{i}=\Lambda_{\alpha_{i}},
$$

and let $u_{i} \in H^{1}(\Omega)$ denote a weak solution to the equation

$$
\operatorname{div}\left(A_{i} \nabla u_{i}\right)=0 \text { in } \Omega .
$$

Applying (2.9) to $f=\left.u_{i}\right|_{\partial \Omega}$ and $g=\left.u_{3-i}\right|_{\partial \Omega}$, we get

and

$$
\left\langle\Lambda_{1} u_{1}, u_{2}\right\rangle=\int_{\Omega} A_{1} \nabla u_{1} \cdot \nabla u_{2} d x
$$

$$
\left\langle\Lambda_{2} u_{2}, u_{1}\right\rangle=\int_{\Omega} A_{2} \nabla u_{2} \cdot \nabla u_{1} d x
$$

which yields

$$
\left\langle\left(\Lambda_{1}-\Lambda_{2}\right) u_{1}, u_{2}\right\rangle=\int_{\Omega}\left(A_{1}-A_{2}\right) \nabla u_{1} \cdot \nabla u_{2} d x,
$$

by (2.10). 


\section{Can we determine the unknown function $\alpha$ from $\Lambda_{\alpha}$ ?}

In this section we examine the inverse problem of identifying $\alpha$ from the knowledge of $\Lambda_{\alpha}$.

Analysis of the problem for general unknown functions. Let $\gamma$ be a nonempty open subset of $\partial \omega$. We put

$$
\Gamma=\gamma \times(-2 L, 2 L) \text { for some fixed } L>0,
$$

and define the functional space

$$
\widetilde{H}_{\Gamma}^{1 / 2}(\partial \Omega)=\left\{f \in \widetilde{H}^{1 / 2}(\partial \Omega) ; \operatorname{supp} f \subset \Gamma\right\} .
$$

Put

$$
M=\max _{i=1,2}\left\|\alpha_{i}\right\|_{W^{1, \infty}(\mathbb{R})}, \quad \text { for } \alpha_{i} \in W^{1, \infty}(\mathbb{R}), i=1,2 .
$$

We note

$$
A_{i}=A\left(x^{\prime}, \alpha_{i}\left(x_{3}\right)\right)
$$

and

$$
\Lambda_{i}=\Lambda_{\alpha_{i}}
$$

In light of (2.11), we have

$$
\left\langle\left(\Lambda_{1}-\Lambda_{2}\right) u_{1}, u_{2}\right\rangle=\int_{\Omega}\left(A_{1}-A_{2}\right) \nabla u_{1} \cdot \nabla u_{2} d x,
$$

for any function $u_{i} \in H^{1}(\Omega)$ which is a weak solution to the equation

$$
\operatorname{div}\left(A_{i} \nabla u_{i}\right)=0 \quad \text { in } \Omega, i=1,2 .
$$

Putting

$$
\Omega^{L}=\omega \times(-L, L),
$$

and assuming that

$$
\alpha_{1}\left(x_{3}\right)=\alpha_{2}\left(x_{3}\right), \quad\left|x_{3}\right|>L,
$$

we may rewrite (3.2) as

$$
\left\langle\left(\Lambda_{1}-\Lambda_{2}\right) u_{1}, u_{2}\right\rangle=\int_{\Omega^{L}}\left(A_{1}-A_{2}\right) \nabla u_{1} \cdot \nabla u_{2} d x .
$$

The following analysis is essentially based on the method built in [3] in the case of bounded domains, that will be adapted to the case of the infinite waveguide $\Omega$ under consideration. To this purpose we set

$$
\Gamma_{\rho}=\{x \in \Gamma ; \operatorname{dist}(x, \partial \Gamma)>\rho\} \quad \text { and } \quad U_{\rho}=\left\{x \in \mathbb{R}^{3} ; \operatorname{dist}\left(x, \Gamma_{\rho}\right)<\rho / 4\right\},
$$


for all $\rho \in\left(0, \rho_{0}\right]$, where $\rho_{0}$ is some characteristic constant depending only on $\omega$ and $L$, which is defined in [3]. Upon eventually shortening $\rho_{0}$, we can assume without loss of generality that $\Gamma_{0}=\gamma_{0} \times[-L, L] \subset \Gamma_{\rho}$ for some $\gamma_{0} \Subset \gamma$. In view of [3], we thus may find a Lipschitz domain $\Omega_{\rho}$ satisfying simultaneously

$$
\begin{aligned}
& \Omega \subset \Omega_{\rho}, \\
& \Gamma_{0} \subset \partial \Omega \cap \Omega_{\rho} \Subset \Gamma,
\end{aligned}
$$

and

$$
\operatorname{dist}\left(x, \partial \Omega_{\rho}\right) \geq \rho / 2, \quad \text { for all } x \in U_{\rho} .
$$

Moreover we know from [2, Section 3] that there is a unitary $C^{\infty}$ vector field $\tilde{v}$, defined in some suitable neighborhood of $\partial \omega \times(-2 L, 2 L)$, which is non tangential to $\partial \Omega$ and points to the exterior of $\Omega$. For $x^{0} \in \overline{\Gamma_{\rho}}$ we pick $\tau_{0}>0$ sufficiently small so the point $z_{\tau}=x^{0}+\tau \tilde{v}\left(x^{0}\right)$ obeys $C \tau \leq \operatorname{dist}\left(z_{\tau}, \partial \Omega\right) \leq \tau$ for every $\tau \in\left(0, \tau_{0}\right]$, by [2, Lemma 3.3]. Here $\tau_{0}$ and $C$ are two positive constants depending only on $\Omega$, and $\lambda, \underline{t}$ and $\bar{t}$ are the same as in (2.3).

In light of [16], the operator $\operatorname{div}\left(A_{i} \nabla \cdot\right), i=1,2$, has a Dirichlet Green function $G_{i}=G_{i}(x, y)$ on $\Omega_{\rho}$. More specifically, $G_{i}(x)=G_{i}\left(x, z_{\tau}\right)$ is the solution to the BVP

$$
\left\{\begin{array}{ll}
\operatorname{div}\left(A_{i} \nabla G_{i}\right)=-\delta\left(x-z_{\tau}\right) & \text { in } \mathcal{D}^{\prime}\left(\Omega_{\rho}\right) \\
G_{i}=0 & \text { on } \partial \Omega_{\rho},
\end{array} \quad i=1,2,\right.
$$

and we will see in the coming lemma that the claim of [3, Corollary 3.4] remains essentially unchanged for the unbounded domain $\Omega_{\rho}$ arising in this framework.

Lemma 3.1. There are two constants

$$
\tau_{0}=\tau_{0}(\Omega, \rho)>0 \quad \text { and } \quad C=C(\Omega, \lambda, \underline{t}, \bar{t}),
$$

such that the restriction $G_{i}\left(., z_{\tau}\right)$ to $\Omega, i=1,2$, belongs to $H^{1}(\Omega)$, and satisfies

$$
\left\|G_{i}\left(\cdot, z_{\tau}\right)\right\|_{H^{1}(\Omega)} \leq C \tau^{-1 / 2}, \quad 0<\tau \leq \tau_{0} .
$$

Proof. Let the space $Y^{2,1}(\Omega)=\left\{u \in L^{6}(\Omega) ; \nabla u \in L^{2}(\Omega)^{3}\right\}$ be endowed with the norm $\|v\|_{Y^{2,1}(\Omega)}=\|v\|_{L^{6}(\Omega)}+\|\nabla v\|_{L^{2}(\Omega)^{3}}$ and choose $\tau_{0}=\tau_{0}(\Omega, \rho)$ so small relative to $\rho$ that $\operatorname{dist}\left(z_{\tau}, \partial \Omega_{\rho}\right)>\tau$ for all $\tau \in\left(0, \tau_{0}\right]$. Since the ball $B_{\tau / 3}\left(z_{\tau}\right)$ centered at $z_{\tau}$ with radius $\tau / 3$, is embedded in $\Omega_{\rho} \backslash \bar{\Omega}$, we have

$$
\left\|G_{i}\left(\cdot, z_{\tau}\right)\right\|_{Y^{2,1}(\Omega)} \leq\left\|G_{i}\left(\cdot, z_{\tau}\right)\right\|_{Y^{2,1}\left(\Omega_{\rho} \backslash B_{\tau / 3}\left(z_{\tau}\right)\right)} \leq C \tau^{-1 / 2}, \quad 0<\tau \leq \tau_{0},
$$


directly from [16, Formula (4.45)]. Here $C$ is some positive constant depending only on $\Omega, \lambda, \underline{t}$ and $\bar{t}$.

Finally, since the Poincaré inequality holds in $\Omega$ because $\omega$ is bounded, we may deduce (3.6) from (3.7).

Further, as a Green function is a Levi function ${ }^{2}$, it behaves locally like a parametrix. Hence, by applying [30, Formula (8.4)], $G_{i}$ can be brought into the form $G_{i}(x)=C\left(\operatorname{det}\left(A_{i}\left(z_{\tau}\right)\right)\right)^{-1 / 2}\left(A_{i}\left(z_{\tau}\right)^{-1}\left(x-z_{\tau}\right) \cdot\left(x-z_{\tau}\right)\right)^{-1 / 2}+R_{i}(x), \quad i=1,2$,

where $A_{i}\left(z_{\tau}\right)$ is a shorthand for $A\left(z_{\tau}^{\prime}, \alpha_{i}\left(\left(z_{\tau}\right)_{3}\right)\right), C>0$ is a constant, and the reminder $R_{i}$ obeys the conditions

$$
\begin{aligned}
& \text { there exists }\left(r_{0}, \alpha\right) \in \mathbb{R}_{+}^{*} \times(0,1) \text {, such that } \\
& \left|R_{i}(x)\right|+\left|x-z_{\tau}\right|\left|\nabla R_{i}\right| \leq C\left|x-z_{\tau}\right|^{-1+\alpha}, \quad x \in \Omega_{\rho},\left|x-z_{\tau}\right| \leq r_{0} .
\end{aligned}
$$

On the other hand it holds true for $i=1,2$, that $\operatorname{div}\left(A_{i} \nabla G_{i}\right)=0$ in the weak sense in $\Omega$, so (3.4) entails

$$
\left\langle\left(\Lambda_{1}-\Lambda_{2}\right) G_{1}, G_{2}\right\rangle=\int_{\Omega^{L}}\left(A_{1}-A_{2}\right) \nabla G_{1} \cdot \nabla G_{2} d x .
$$

Taking into account that $\left.G_{i}\right|_{\partial \Omega} \in \widetilde{H}^{1 / 2}(\partial \Omega)$, this yields

$$
\left|\int_{\Omega^{L}}\left(A_{1}-A_{2}\right) \nabla G_{1} \cdot \nabla G_{2} d x\right| \leq\left\|\Lambda_{1}^{\Gamma}-\Lambda_{2}^{\Gamma}\right\|\left\|G_{1}\right\|_{\widetilde{H}^{1 / 2}(\partial \Omega)}\left\|G_{2}\right\|_{\tilde{H}^{1 / 2}(\partial \Omega)},
$$

where $\Lambda_{i}^{\Gamma}$ is the restriction of $\Lambda_{i}$ to the closed subspace $\widetilde{H}_{\Gamma}^{1 / 2}(\partial \Omega)$, and consequently

$$
\left|\int_{\Omega^{L}}\left(A_{1}-A_{2}\right) \nabla G_{1} \cdot \nabla G_{2} d x\right| \leq C\left\|\Lambda_{1}^{\Gamma}-\Lambda_{2}^{\Gamma}\right\|\left\|G_{1}\right\|_{H^{1}(\Omega)}\left\|G_{2}\right\|_{H^{1}(\Omega)} .
$$

The next step involves picking $x^{0} \in \Gamma_{0}$ such that

$$
\left|\alpha_{1}\left(x_{3}^{0}\right)-\alpha_{2}\left(x_{3}^{0}\right)\right|=\left\|\alpha_{1}-\alpha_{2}\right\|_{L^{\infty}(-L, L)} .
$$

${ }^{2}$ Let $E=\sum_{1 \leq i, j \leq m} \alpha_{i j}(x) \partial_{i j}^{2}$ be an elliptic operator acting in a subdomain of $\mathbb{R}^{m}, m \geq 3$, and let $\beta(x)=\left(\beta_{i j}(x)\right)_{1 \leq i, j \leq m}$ be the inverse matrix to $\alpha(x)=\left(\alpha_{i j}(x)\right)_{1 \leq i, j \leq m}$. Then $H(x, y)=\left|\mathbb{S}^{m-1}\right|^{-1}|\operatorname{det}(\alpha(y))|^{-1 / 2}[\beta(y)(x-y) \cdot(x-y)]^{\frac{2-m}{2}}$ is solution to the equation $\sum_{1 \leq i, j \leq m} \alpha_{i j}(y) \partial_{i j}^{2} H(\cdot, y)=0$, and is called a parametrix of $E$. With reference to [30], any function $L=L(x, y)$ which is smooth outside $x \neq y$ and satisfies $\partial^{\alpha}(L-H)(x, y)=$ $O\left(|x-y|^{2+\lambda-|\alpha|-m}\right)$ for some $\lambda>0$ and any $\alpha \in \mathbb{N}^{m},|\alpha| \leq 2$, is a Levi function. 
We may actually assume without loss of generality that

$$
\left|\alpha_{1}\left(x_{3}^{0}\right)-\alpha_{2}\left(x_{3}^{0}\right)\right|=\alpha_{1}\left(x_{3}^{0}\right)-\alpha_{2}\left(x_{3}^{0}\right),
$$

hence

$$
\alpha_{1}\left(x_{3}^{0}\right)-\alpha_{2}\left(x_{3}^{0}\right)=\left\|\alpha_{1}-\alpha_{2}\right\|_{L^{\infty}(-L, L)} .
$$

Let us next decompose the left hand side of (3.9) into the sum

$$
\begin{aligned}
\int_{\Omega^{L}}\left(A_{1}-A_{2}\right) \nabla G_{1} \cdot \nabla G_{2} d x= & \int_{\Omega^{L} \cap B\left(z_{\tau}, \rho\right)}\left(A_{1}-A_{2}\right) \nabla G_{1} \cdot \nabla G_{2} d x \\
& +\int_{\Omega^{L} \backslash B\left(z_{\tau}, \rho\right)}\left(A_{1}-A_{2}\right) \nabla G_{1} \cdot \nabla G_{2} d x,
\end{aligned}
$$

where, as previously stated, $z_{\tau}=x^{0}+\tau \tilde{v}\left(x^{0}\right)$ for some $\tau \in \mathbb{R}_{+}^{*}$. Recalling (3.8) we see that the first term in the right hand side of (3.11) is lower bounded by

$$
\begin{aligned}
& \int_{B\left(z_{\tau}, \rho\right) \cap \Omega^{L}} \frac{A_{2}\left(z_{\tau}\right)^{-1}\left(A_{1}(x)-A_{2}(x)\right) A_{1}\left(z_{\tau}\right)^{-1}\left(x-z_{\tau}\right) \cdot\left(x-z_{\tau}\right)}{\mathfrak{D}} d x \\
& \quad-c \int_{\Omega^{L} \cap B\left(z_{\tau}, \rho\right)}\left|x-z_{\tau}\right|^{-4+2 \alpha} d x,
\end{aligned}
$$

where

$$
\mathfrak{D}=\left(A_{1}\left(z_{\tau}\right)^{-1} \cdot\left(x-z_{\tau}\right)\right)^{3 / 2}\left(A_{2}\left(z_{\tau}\right)^{-1} \cdot\left(x-z_{\tau}\right)\right)^{3 / 2},
$$

and where $c$ is a positive constant depending only on $M$ and $\omega$. From this and the Lipschitz continuity of

$$
x \longmapsto A_{i}(x), \quad i=1,2,
$$

then follows upon writing $A_{i}\left(x^{0}\right)$ instead of $A\left(\left(x^{0}\right)^{\prime}, \alpha_{i}\left(x_{3}^{0}\right)\right)$, that

$$
\begin{aligned}
& \int_{\Omega^{L} \cap B\left(z_{\tau}, \rho\right)}\left(A_{1}-A_{2}\right) \nabla G_{1} \cdot \nabla G_{2} d x \\
& \geq \int_{B\left(z_{\tau}, \rho\right) \cap \Omega^{L}} \frac{\left.\left(A_{1}\left(x^{0}\right)^{-1}\right)-A_{2}\left(x^{0}\right)^{-1}\right)\left(x-z_{\tau}\right) \cdot\left(x-z_{\tau}\right)}{\mathfrak{D}} d x \\
& \quad-c \int_{\Omega^{L} \cap B\left(z_{\tau}, \rho\right)}\left|x-z_{\tau}\right|^{-4}\left(\left|x-x^{0}\right|^{2 \alpha}+\left|x^{0}-z_{\tau}\right|^{2 \alpha}\right) d x .
\end{aligned}
$$

On the other hand, since

$$
\left|\int_{\Omega^{L} \backslash B\left(z_{\tau}, \rho\right)}\left(A_{1}-A_{2}\right) \nabla G_{1} \cdot \nabla G_{2} d x\right|
$$


is majorized by, say, $c$, and

$$
\left\|\Lambda_{1}^{\Gamma}-\Lambda_{2}^{\Gamma}\right\|\left\|G_{1}\right\|_{H^{1}(\Omega)}\left\|G_{2}\right\|_{H^{1}(\Omega)} \leq C \tau^{-1}\left\|\Lambda_{1}^{\Gamma}-\Lambda_{2}^{\Gamma}\right\|,
$$

by Lemma 3.1, we deduce from (3.11) and (3.12) that

$$
\begin{aligned}
& \int_{B\left(z_{\tau}, \rho\right) \cap \Omega^{L}} \frac{\left(A_{1}\left(x^{0}\right)^{-1}-A_{2}\left(x^{0}\right)^{-1}\right)\left(x-z_{\tau}\right) \cdot\left(x-z_{\tau}\right)}{\mathfrak{D}} d x \\
& \quad \leq C\left(\tau^{-1+\alpha}+1+\tau^{-1}\left\|\Lambda_{1}^{\Gamma}-\Lambda_{2}^{\Gamma}\right\|\right) .
\end{aligned}
$$

The main ingredient in the analysis developed in [3] is the ellipticity condition [3, Formula (2.5)] imposed on $\partial_{t} A\left(x^{\prime}, t\right)$, implying

$$
\begin{aligned}
& \int_{B\left(z_{\tau}, \rho\right) \cap \Omega^{L}} \frac{\left(A_{1}\left(x^{0}\right)^{-1}-A_{2}\left(x^{0}\right)^{-1}\right)\left(x-z_{\tau}\right) \cdot\left(x-z_{\tau}\right)}{\mathfrak{D}} d x \\
& \quad \geq C_{0} \tau^{-1}\left(\alpha_{1}\left(x_{3}^{0}\right)-\alpha_{2}\left(x_{3}^{0}\right)\right),
\end{aligned}
$$

for some constant $C_{0}>0$. This, (3.10) and (3.13) finally lead to the desired stability estimate:

$$
\left\|\alpha_{1}-\alpha_{2}\right\|_{L^{\infty}(-L, L)} \leq C\left\|\Lambda_{1}^{\Gamma}-\Lambda_{2}^{\Gamma}\right\| .
$$

Unfortunately, it turns out that [3, Formula (2.5)] is not fulfilled by $\partial_{t} A\left(x^{\prime}, t\right)$ in this framework. This can be seen from the following explicit expression

$$
\begin{aligned}
& \lambda_{1}\left(x^{\prime}, t\right)=0, \\
& \lambda_{2}\left(x^{\prime}, t\right)=\left|x^{\prime}\right|^{2} t-\sqrt{\left|x^{\prime}\right|^{4} t^{2}+\left|x^{\prime}\right|^{2}},
\end{aligned}
$$

and

$$
\lambda_{3}\left(x^{\prime}, t\right)=\left|x^{\prime}\right|^{2} t+\sqrt{\left|x^{\prime}\right|^{4} t^{2}+\left|x^{\prime}\right|^{2}},
$$

of the eigenvalues of $\partial_{t} A\left(x^{\prime}, t\right)$, showing that the spectrum of $\partial_{t} A\left(x^{\prime}, t\right)$ has a negative component for $x^{\prime} \in \partial \omega$. Moreover, due to the occurrence of this negative eigenvalue, the weak monotonicity assumption [2, Formula (5.7)] is not satisfied by the conductivity matrix under consideration either. Therefore, the approach developed in [3] does not apply to the inverse problem of determining $\alpha$ from the knowledge of $\Lambda_{\alpha}$, which remains open in the general case.

Nevertheless, we will see in Section 4 that this is not the case for constant unknown functions anymore. But, prior to examining this peculiar framework, we will now deduce from the above reasoning, upon substituting a suitable matrix $A^{\bullet}$ for $A$, that unknown functions $\alpha$ which are close to some a priori fixed constant value may well be identified from the associated DN map. 
The case of unknown functions close to a constant value. Put

$$
A^{\bullet}\left(x^{\prime}, t\right)=t\left(\begin{array}{ccc}
1+x_{2}^{2} & -x_{2} x_{1} & -x_{2} \\
-x_{2} x_{1} & 1+x_{1}^{2} & x_{1} \\
-x_{2} & x_{1} & 1
\end{array}\right), \quad x^{\prime} \in \omega, t \in \mathbb{R},
$$

so that we have

$$
A^{\bullet}\left(x^{\prime}, t\right)=t A^{\bullet}\left(x^{\prime}, 1\right) .
$$

Define

$$
\Lambda_{\alpha}^{\bullet}: f \longmapsto A^{\bullet} \nabla u \cdot v,
$$

i.e. the map obtained from $\Lambda_{\alpha}$ by replacing $A\left(x^{\prime}, \alpha\left(x_{3}\right)\right)$ by $A^{\bullet}\left(x^{\prime}, \alpha\left(x_{3}\right)\right)$. Then, by arguing as in the derivation of (2.11), we obtain that

$$
\left\langle\left(\Lambda_{\alpha}-\Lambda_{\alpha}^{\bullet}\right) u, u^{\bullet}\right\rangle=\int_{\Omega}\left(A\left(x^{\prime}, \alpha\left(x_{3}\right)\right)-A^{\bullet}\left(x^{\prime}, \alpha\left(x_{3}\right)\right) \nabla u \cdot \nabla u^{\bullet} d x,\right.
$$

for all $u, u^{\bullet} \in H^{1}(\Omega)$ satisfying

$$
\operatorname{div}\left(A\left(x^{\prime}, \alpha\left(x_{3}\right)\right) \nabla u\right)=\operatorname{div}\left(A^{\bullet}\left(x^{\prime}, \alpha\left(x_{3}\right)\right) \nabla u^{\bullet}\right)=0
$$

in the weak sense in $\Omega$. By direct calculation we note from the definitions of $A$ and $A^{\bullet}$ that

$$
A(x, t)-A^{\bullet}\left(x^{\prime}, t\right)=(1-t)\left(B_{0}-t B_{1}\left(x^{\prime}\right)\right), \quad\left(x^{\prime}, t\right) \in \omega \times \mathbb{R},
$$

where $B_{0}$ stands for the identity matrix of $\mathbb{R}^{3}$ and

$$
B_{1}\left(x^{\prime}\right)=\left(\begin{array}{ccc}
x_{2}^{2} & -x_{2} x_{1} & 0 \\
-x_{2} x_{1} & x_{1}^{2} & 0 \\
0 & 0 & 0
\end{array}\right), \quad x^{\prime} \in \omega
$$

Assume that

$$
\alpha \in L^{\infty}(\mathbb{R}), \quad\|\alpha-1\|_{L^{\infty}(\mathbb{R})} \leq M .
$$

Given $f$ (resp., $g$ ) in $\tilde{H}^{1 / 2}(\partial \Omega)$, we denote $u$ (resp., $u^{\bullet}$ ) the solution to equation (1.3) (resp., equation (1.3), where $\left(A^{\bullet}, g\right)$ is substituted to $\left.(A, f)\right)$. Applying (3.16), we get that

$$
\left|\left\langle\left(\Lambda_{\alpha}-\Lambda_{\alpha}^{\bullet}\right) f, g\right\rangle\right| \leq c(M, \omega)\|\alpha-1\|_{\infty}\|u\|_{H^{1}(\Omega)}\left\|u^{\bullet}\right\|_{H^{1}(\Omega)},
$$

where $C=C(M, \omega)$ is some positive constant depending only on $M$ and $\omega$. Therefore we have

$$
\left|\left\langle\left(\Lambda_{\alpha}-\Lambda_{\alpha}^{\bullet}\right) f, g\right\rangle\right| \leq C\|\alpha-1\|_{\infty}\|f\|_{\tilde{H}^{1 / 2}(\partial \Omega)}\|g\|_{\tilde{H}^{1 / 2}(\partial \Omega)},
$$


by (2.5), hence

$$
\left\|\Lambda_{\alpha}-\Lambda_{\alpha}^{\bullet}\right\|_{\mathcal{L}\left(\widetilde{H}^{1 / 2}(\partial \Omega), \widetilde{H}^{-1 / 2}(\partial \Omega)\right)} \leq C\|\alpha-1\|_{\infty},
$$

showing that $\Lambda_{\alpha}^{\bullet}$ is a suitable approximation of $\Lambda_{\alpha}$ provided $\alpha$ is sufficiently close to $1 .{ }^{3}$

Actually, the main benefit of dealing with $\Lambda_{\alpha}^{\bullet}$ instead of $\Lambda_{\alpha}$ in the inverse problem of determining $\alpha$ from $\Lambda_{\alpha}$, boils down to the following identity

$$
\partial_{t} A^{\bullet}\left(x^{\prime}, t\right)=A^{\bullet}\left(x^{\prime}, 1\right)=A\left(x^{\prime}, 1\right), \quad \text { for all } x^{\prime} \in \omega \text { and } t \in \mathbb{R},
$$

ensuring that the ellipticity condition [3, Formula (2.5)] required by the method presented in [3], is verified by $A^{\bullet}$. Now, in light of the reasoning developed in the first part of this section, which is borrowed from the proof of [3, Theorem 2.2], we derive the following theorem.

Theorem 3.1. For $L>0, \underline{t}>0$ and $M>0$ fixed, let $\alpha_{i} \in W^{1, \infty}(\mathbb{R}), i=1,2$, obey (3.3) and fulfill $\alpha_{i} \geq \underline{t}$ and $\left\|\alpha_{i}\right\|_{W^{1, \infty}(\mathbb{R})} \leq M$. Then there exists a constant $C>0$ depending only on $\omega, M$ and $L$, such that we have

$$
\left\|\alpha_{1}-\alpha_{2}\right\|_{L^{\infty}(\mathbb{R})} \leq C\left\|\left(\Lambda_{\alpha_{1}}^{\bullet}\right)^{\Gamma}-\left(\Lambda_{\alpha_{2}}^{\bullet}\right)^{\Gamma}\right\|_{\mathcal{L}\left(\widetilde{H}^{1 / 2}(\partial \Omega), \widetilde{H}^{-1 / 2}(\partial \Omega)\right)},
$$

where $\left(\Lambda_{\alpha_{i}}^{\bullet}\right)^{\Gamma}=\left(\Lambda_{\alpha_{i}}^{\bullet}\right)_{\mid \tilde{H}_{\Gamma}^{1 / 2}(\partial \Omega)}$ for $i=1,2$, the space $\widetilde{H}_{\Gamma}^{1 / 2}(\partial \Omega)$ being the same as in (3.1).

\section{The case of constant unknown functions}

In this section we address the case of affine twisting functions $\theta$, that is constant functions $\alpha$, by means of the partial Fourier transform $\mathcal{F}_{x_{3}}$ with respect to the variable $x_{3}$. This is suggested by the translational invariance of the system under consideration in the infinite direction $x_{3}$, arising from the fact that the matrix $A\left(x^{\prime}, \alpha\left(x_{3}\right)\right)$ appearing in (1.3) does not depend on $x_{3}$ in this peculiar case.

In the sequel, we note $\xi$ the Fourier variable associated with $x_{3}$ and we write $\hat{w}$ instead of $\mathcal{F}_{x_{3}} w$ for every function $w=w\left(x^{\prime}, x_{3}\right)$ :

$$
\hat{w}\left(x^{\prime}, \xi\right)=\left(\mathcal{F}_{x_{3}} w\right)\left(x^{\prime}, \xi\right), \quad x^{\prime} \in \omega, \xi \in \mathbb{R} .
$$

The first step of the method is to re-express the system (1.3) in the Fourier plane $\left\{\left(x^{\prime}, \xi\right), x^{\prime} \in \omega, \xi \in \mathbb{R}\right\}$.

${ }^{3}$ Note that 1 can be replaced by any constant $\mu \neq 0$ upon substituting $A^{\bullet}\left(\mu x^{\prime}, \frac{t}{\mu}\right)$ for $A^{\bullet}\left(x^{\prime}, t\right)$ in the above reasoning, since $A(x, t)=A\left(\mu x^{\prime}, \frac{t}{\mu}\right)$. 
Rewriting the BVP in the Fourier variables. We start with two useful technical lemmas.

Lemma 4.1. For every $w \in H^{1}(\Omega)$ the identity

$$
\widehat{\partial_{x_{j}} w}=\partial_{x_{j}} \hat{w}
$$

holds for $j=1,2$.

Proof. Fix $j=1$, 2. For every $\varphi \in C_{0}^{\infty}(\omega)$ and $\psi \in \mathcal{S}(\mathbb{R})$, we have

$$
\begin{aligned}
& \int_{\omega} \varphi\left(x^{\prime}\right)\left(\int_{\mathbb{R}} \partial_{x_{j}} w\left(x^{\prime}, x_{3}\right) \hat{\psi}\left(x_{3}\right) d x_{3}\right) d x^{\prime} \\
& =\int_{\mathbb{R}} \hat{\psi}\left(x_{3}\right)\left(\int_{\omega} \partial_{x_{j}} w\left(x^{\prime}, x_{3}\right) \varphi\left(x^{\prime}\right) d x^{\prime}\right) d x_{3},
\end{aligned}
$$

from Fubini's theorem. By integrating by parts in the last integral, we obtain

$$
\int_{\omega} \partial_{x_{j}} w\left(x^{\prime}, x_{3}\right) \varphi\left(x^{\prime}\right) d x^{\prime}=-\int_{\omega} w\left(x^{\prime}, x_{3}\right) \partial_{x_{j}} \varphi\left(x^{\prime}\right) d x^{\prime}, \quad \text { a.e. } x_{3} \in \mathbb{R},
$$

so we get

$$
\begin{aligned}
& \int_{\omega} \varphi\left(x^{\prime}\right)\left(\int_{\mathbb{R}} \partial_{x_{j}} w\left(x^{\prime}, x_{3}\right) \hat{\psi}\left(x_{3}\right) d x_{3}\right) d x^{\prime} \\
& =-\int_{\mathbb{R}} \hat{\psi}\left(x_{3}\right)\left(\int_{\omega} w\left(x^{\prime}, x_{3}\right) \partial_{x_{j}} \varphi\left(x^{\prime}\right) d x^{\prime}\right) d x_{3} \\
& =-\int_{\omega} \partial_{x_{j}} \varphi\left(x^{\prime}\right)\left(\int_{\mathbb{R}} w\left(x^{\prime}, x_{3}\right) \hat{\psi}\left(x_{3}\right) d x_{3}\right) d x^{\prime} .
\end{aligned}
$$

Further, the operator $\mathcal{F}_{x_{3}}$ being selfadjoint in $L^{2}(\mathbb{R})$, it is true that

$$
\int_{\mathbb{R}} w\left(x^{\prime}, x_{3}\right) \hat{\psi}\left(x_{3}\right) d x_{3}=\int_{\mathbb{R}} \hat{w}\left(x^{\prime}, \xi\right) \psi(\xi) d \xi, \quad \text { a.e. } x^{\prime} \in \omega,
$$

whence

$$
\begin{gathered}
\int_{\omega} \varphi\left(x^{\prime}\right)\left(\int_{\mathbb{R}} \partial_{x_{j}} w\left(x^{\prime}, x_{3}\right) \hat{\psi}\left(x_{3}\right) d x_{3}\right) d x^{\prime} \\
=-\int_{\omega} \partial_{x_{j}} \varphi\left(x^{\prime}\right)\left(\int_{\mathbb{R}} \hat{w}\left(x^{\prime}, \xi\right) \psi(\xi) d \xi\right) d x^{\prime} \\
=\int_{\omega} \varphi\left(x^{\prime}\right)\left(\int_{\mathbb{R}} \partial_{x_{j}} \hat{w}\left(x^{\prime}, \xi\right) \psi(\xi) d \xi\right) d x^{\prime},
\end{gathered}
$$


by integrating by parts. From the density of $C_{0}^{\infty}(\omega)$ in $L^{2}(\omega)$, the above identity entails that

$$
\int_{\mathbb{R}} \partial_{x_{j}} w\left(x^{\prime}, x_{3}\right) \hat{\psi}\left(x_{3}\right) d x_{3}=\int_{\mathbb{R}} \partial_{x_{j}} \hat{w}\left(x^{\prime}, \xi\right) \psi(\xi) d \xi \text {, a.e. } x^{\prime} \in \omega,
$$

for every $\psi \in \mathcal{S}(\mathbb{R})$. From this, the selfadjointness of $\mathcal{F}_{x_{3}}$ and the density of $\mathcal{S}(\mathbb{R})$ in $L^{2}(\mathbb{R})$, then follows that $\widehat{\partial_{x_{j}} w}=\partial_{x_{j}} \hat{w}$.

Lemma 4.2. Let $C=\left(C_{k l}\right)_{1 \leq k, l \leq 3} \in W^{1, \infty}(\omega)^{3 \times 3}$ be such that $\left(C_{k l}\left(x^{\prime}\right)\right)_{1 \leq k, l \leq 3}$ is symmetric for all $x^{\prime} \in \omega$. Then every $w \in H^{1}(\Omega)$ obeying

$$
\int_{\Omega} C \nabla w \cdot \nabla v d x=0 \quad \text { for all } v \in H_{0}^{1}(\Omega),
$$

satisfies the equation

$$
-\operatorname{div}_{x^{\prime}}\left(\widetilde{C}\left(x^{\prime}\right) \nabla_{x^{\prime}} \hat{w}\right)+P\left(x^{\prime}, \xi\right) \cdot \nabla_{x^{\prime}} \hat{w}+q\left(x^{\prime}, \xi\right) \hat{w}=0 \text { in } \mathcal{D}^{\prime}(\Omega),
$$

with

$$
\begin{aligned}
\widetilde{C}\left(x^{\prime}\right) & =\left(C_{i j}\left(x^{\prime}\right)\right)_{1 \leq i, j \leq 2} \\
P\left(x^{\prime}, \xi\right) & =-i 2 \xi\left(\begin{array}{l}
C_{31}\left(x^{\prime}\right) \\
C_{32}\left(x^{\prime}\right)
\end{array}\right)
\end{aligned}
$$

and

$$
q\left(x^{\prime}, \xi\right)=-i \xi \operatorname{div}_{x^{\prime}}\left(\begin{array}{l}
C_{31}\left(x^{\prime}\right) \\
C_{32}\left(x^{\prime}\right)
\end{array}\right)+\xi^{2} C_{33}\left(x^{\prime}\right), \quad\left(x^{\prime}, \xi\right) \in \Omega .
$$

Moreover, if $w \in H^{2}(\Omega)$ is solution to (4.1) then (4.2) holds for a.e. $\left(x^{\prime}, \xi\right) \in \Omega$.

Proof. Choose $v=\varphi \otimes \hat{\psi}$ in (4.1), with $\varphi \in C_{0}^{\infty}(\omega)$ and $\psi \in \mathcal{S}(\mathbb{R})$, so we have

$$
\sum_{k, l=1,2,3} \int_{\Omega} C_{k l}\left(x^{\prime}\right) \partial_{x_{k}} w\left(x^{\prime}, x_{3}\right) \partial_{x_{l}}(\varphi \otimes \hat{\psi})\left(x^{\prime}, x_{3}\right) d x^{\prime} d x_{3}=0 .
$$

For $1 \leq k, l \leq 2$, we note that

$$
\begin{gathered}
\int_{\Omega} C_{k l}\left(x^{\prime}\right) \partial_{x_{l}} w\left(x^{\prime}, x_{3}\right) \partial_{x_{k}}(\varphi \otimes \hat{\psi})\left(x^{\prime}, x_{3}\right) d x^{\prime} d x_{3} \\
=\int_{\omega} C_{k l}\left(x^{\prime}\right) \partial_{x_{k}} \varphi\left(x^{\prime}\right)\left(\int_{\mathbb{R}} \partial_{x_{l}} w\left(x^{\prime}, x_{3}\right) \hat{\psi}\left(x_{3}\right) d x_{3}\right) d x^{\prime} \\
=\int_{\Omega} C_{k l}\left(x^{\prime}\right) \partial_{x_{l}} \hat{w}\left(x^{\prime}, \xi\right) \partial_{x_{k}}(\varphi \otimes \psi)\left(x^{\prime}, \xi\right) d x^{\prime} d \xi,
\end{gathered}
$$


directly from Lemma 4.1. Further, the identity

$$
\begin{aligned}
& \int_{\Omega} C_{3 l}\left(x^{\prime}\right) \partial_{x_{l}} w\left(x^{\prime}, x_{3}\right) \partial_{x_{3}}(\varphi \otimes \hat{\psi})\left(x^{\prime}, x_{3}\right) d x^{\prime} d x_{3} \\
& =\int_{\omega} C_{3 j}\left(x^{\prime}\right) \varphi\left(x^{\prime}\right)\left(\int_{\mathbb{R}} \partial_{x_{l}} w\left(x^{\prime}, x_{3}\right) \hat{\psi}^{\prime}\left(x_{3}\right) d x_{3}\right) d x^{\prime} \\
& =\int_{\omega} C_{3 l}\left(x^{\prime}\right) \varphi\left(x^{\prime}\right)\left(\int_{\mathbb{R}} \partial_{x_{l}} w\left(x^{\prime}, x_{3}\right) \widehat{(-i \xi) \psi}\left(x_{3}\right) d x_{3}\right) d x^{\prime}
\end{aligned}
$$

holds for $l=1,2$, so we have

$$
\begin{aligned}
& \int_{\Omega} C_{3 l}\left(x^{\prime}\right) \partial_{x_{l}} w\left(x^{\prime}, x_{3}\right) \partial_{x_{3}}(\varphi \otimes \hat{\psi})\left(x^{\prime}, x_{3}\right) d x^{\prime} d x_{3} \\
& \quad=\int_{\Omega} C_{3 l}\left(x^{\prime}\right)(-i \xi) \partial_{x_{l}} \hat{w}\left(x^{\prime}, \xi\right)(\varphi \otimes \psi)\left(x^{\prime}, \xi\right) d x^{\prime} d \xi
\end{aligned}
$$

Next, since

$$
\begin{aligned}
& \int_{\Omega} C_{k 3}\left(x^{\prime}\right) \partial_{x_{3}} w\left(x^{\prime}, x_{3}\right) \partial_{x_{k}}(\varphi \otimes \hat{\psi})\left(x^{\prime}, x_{3}\right) d x^{\prime} d x_{3} \\
& =\int_{\omega} C_{k 3}\left(x^{\prime}\right) \partial_{x_{k}} \varphi\left(x^{\prime}\right)\left(\int_{\mathbb{R}} \partial_{x_{3}} w\left(x^{\prime}, x_{3}\right) \hat{\psi}\left(x_{3}\right) d x_{3}\right) d x^{\prime} \\
& =-\int_{\omega} C_{k 3}\left(x^{\prime}\right) \partial_{x_{k}} \varphi\left(x^{\prime}\right)\left(\int_{\mathbb{R}} w\left(x^{\prime}, x_{3}\right) \hat{\psi}^{\prime}\left(x_{3}\right) d x_{3}\right) d x^{\prime},
\end{aligned}
$$

for each $k=1,2$, with

$$
\begin{aligned}
\int_{\mathbb{R}} w\left(x^{\prime}, x_{3}\right) \hat{\psi}^{\prime}\left(x_{3}\right) d x_{3} & =-\int_{\mathbb{R}} w\left(x^{\prime}, x_{3}\right) \widehat{(i \xi) \psi}\left(x_{3}\right) d x_{3} \\
& =-\int_{\mathbb{R}}(i \xi) \hat{w}\left(x^{\prime}, \xi\right) \psi(\xi) d \xi
\end{aligned}
$$

then

$$
\begin{aligned}
& \int_{\Omega} C_{k 3}\left(x^{\prime}\right) \partial_{x_{3}} w\left(x^{\prime}, x_{3}\right) \partial_{x_{k}}(\varphi \otimes \hat{\psi})\left(x^{\prime}, x_{3}\right) d x^{\prime} d x_{3} \\
& =\int_{\Omega} C_{k 3}\left(x^{\prime}\right)(i \xi) \hat{w}\left(x^{\prime}, \xi\right) \partial_{x_{k}}(\varphi \otimes \psi)\left(x^{\prime}, \xi\right) d x^{\prime} d \xi
\end{aligned}
$$


Integrating by parts in the right hand side of the above identity we get

$$
\begin{aligned}
& \int_{\Omega} C_{k 3}\left(x^{\prime}\right) \partial_{x_{3}} w\left(x^{\prime}, x_{3}\right) \partial_{x_{k}}(\varphi \otimes \hat{\psi})\left(x^{\prime}, x_{3}\right) d x^{\prime} d x_{3} \\
& =\int_{\Omega} C_{k 3}\left(x^{\prime}\right)(-i \xi) \partial_{x_{k}} \hat{w}\left(x^{\prime}, \xi\right)(\varphi \otimes \psi)\left(x^{\prime}, \xi\right) d x^{\prime} d \xi \\
& \quad+\int_{\Omega} \partial_{x_{k}} C_{k 3}\left(x^{\prime}\right)(-i \xi) \hat{w}\left(x^{\prime}, \xi\right)(\varphi \otimes \psi)\left(x^{\prime}, \xi\right) d x^{\prime} d \xi .
\end{aligned}
$$

Further, bearing in mind that

$$
\partial_{x_{3}}(\varphi \otimes \hat{\psi})=\varphi \otimes \widehat{(-i \xi) \psi}
$$

and observing that

$$
\begin{aligned}
\int_{\mathbb{R}} \partial_{x_{3}} w\left(x^{\prime}, x_{3}\right) \widehat{(-i \xi) \psi}\left(x_{3}\right) d x_{3} & =-\int_{\mathbb{R}} w\left(x^{\prime}, x_{3}\right) \widehat{(-i \xi)^{2} \psi}\left(x_{3}\right) d x_{3} \\
& =-\int_{\mathbb{R}} \hat{w}\left(x^{\prime}, \xi\right)(-i \xi)^{2} \psi(\xi) d \xi
\end{aligned}
$$

we find out that

$$
\begin{gathered}
\int_{\Omega} C_{33}\left(x^{\prime}\right) \partial_{x_{3}} w\left(x^{\prime}, x_{3}\right) \partial_{x_{3}}(\varphi \otimes \hat{\psi})\left(x^{\prime}, x_{3}\right) d x^{\prime} d x_{3} \\
=\int_{\Omega} C_{33}\left(x^{\prime}\right)(-i \xi)^{2} \hat{w}\left(x^{\prime}, \xi\right)(\varphi \otimes \psi)\left(x^{\prime}, \xi\right) d x^{\prime} d \xi .
\end{gathered}
$$

Finally, putting (4.3)-(4.7) together, we end up getting that

$$
\left\langle-\operatorname{div}_{x^{\prime}}\left(\widetilde{C}\left(x^{\prime}\right) \nabla_{x^{\prime}} \hat{w}\right)+P\left(x^{\prime}, \xi\right) \cdot \nabla_{x^{\prime}} \hat{w}+q\left(x^{\prime}, \xi\right) \hat{w}, \Phi\right\rangle=0,
$$

with $\Phi \in C_{0}^{\infty}(\omega) \otimes C_{0}^{\infty}(\mathbb{R})$, where $\langle\cdot, \cdot\rangle$ denotes the duality pairing between $C_{0}^{\infty}(\Omega)$ and $\mathcal{D}^{\prime}(\Omega)$. From this and the density of $C_{0}^{\infty}(\omega) \otimes C_{0}^{\infty}(\mathbb{R})$ in $C_{0}^{\infty}(\Omega)$ then follows that

$$
-\operatorname{div}_{x^{\prime}}\left(\tilde{C}\left(x^{\prime}\right) \nabla_{x^{\prime}} \hat{w}\right)+P\left(x^{\prime}, \xi\right) \cdot \nabla_{x^{\prime}} \hat{w}+q\left(x^{\prime}, \xi\right) \hat{w}=0 \quad \text { in } \mathcal{D}^{\prime}(\Omega),
$$

which completes the proof.

Let $a \in \mathbb{R}$ be fixed. We assume in the remaining of this section that $\alpha\left(x_{3}\right)=a$ for all $x_{3} \in \mathbb{R}$. For notational simplicity we write $A_{a}\left(x^{\prime}\right)$ instead of $A\left(x^{\prime}, \alpha\left(x_{3}\right)\right)$. With the help of Lemma 4.2 we will first re-express (1.3) in the Fourier plane. 
For $g \in H^{1}(\mathbb{R}) \cap L^{1}(\mathbb{R})$ such that

$$
\int_{\mathbb{R}} g\left(x_{3}\right) d x_{3}=1
$$

and for $h \in H^{1 / 2}(\partial \omega)$, we consider the $H^{1}(\Omega)$-solution $u$ to (1.3), with

$$
f\left(x^{\prime}, x_{3}\right)=g\left(x_{3}\right) h\left(x^{\prime}\right), x^{\prime} \in \partial \omega, x_{3} \in \mathbb{R} .
$$

Since $u$ is solution to (4.1) with $C=A_{a} \in W^{1, \infty}(\omega)^{3 \times 3}$, we deduce from Lemma 4.2 that $\hat{u} \in L^{2}\left(\mathbb{R} ; H^{1}(\omega)\right)$ is solution to the system

$$
\begin{cases}-\operatorname{div}_{x^{\prime}}\left(\tilde{A}_{a}\left(x^{\prime}\right) \nabla_{x^{\prime}} \hat{u}\left(x^{\prime}, \xi\right)\right)-2 i a \xi x^{\prime \perp} \cdot \nabla_{x^{\prime}} \hat{u}+\xi^{2} \hat{u}=0 & \text { in } \mathcal{D}^{\prime}(\Omega), \\ \hat{u}(\cdot, \xi)=\hat{g}(\xi) f & \text { on } \partial \omega, \\ & \text { for all } \xi \in \mathbb{R}\end{cases}
$$

where

$$
x^{\prime \perp}=\left(-x_{2}, x_{1}\right) \quad \text { and } \quad \tilde{A}_{a}\left(x^{\prime}\right)=\left(\begin{array}{cc}
1+x_{2}^{2} a^{2} & -x_{2} x_{1} a^{2} \\
-x_{2} x_{1} a^{2} & 1+x_{1}^{2} a^{2}
\end{array}\right)
$$

We turn now to examining (4.8).

Analysis of the variational problem associated with (4.8). Let us consider the bilinear form

$$
\begin{aligned}
& \mathcal{A}_{\xi}[(v, w),(\varphi, \psi)] \\
& =\int_{\omega} \tilde{A}_{a} \nabla v \cdot \nabla \varphi d x^{\prime}-2 a \xi \int_{\omega} x^{\perp} \cdot \nabla w \varphi d x^{\prime} \\
& \quad+\xi^{2} \int_{\omega} v \varphi d x^{\prime}+\int_{\omega} \tilde{A}_{a} \nabla w \cdot \nabla \psi d x^{\prime} \\
& \quad+2 a \xi \int_{\omega} x^{\prime \perp} \cdot \nabla v \psi d x^{\prime}+\xi^{2} \int_{\omega} w \psi d x^{\prime}, \quad(v, w),(\varphi, \psi) \in \mathcal{H},
\end{aligned}
$$

defined on the Hilbert space

$$
\mathcal{H}=H_{0}^{1}(\omega) \times H_{0}^{1}(\omega)
$$

endowed with the norm

$$
\|(v, w)\|_{\mathcal{H}}=\left(\|\nabla v\|_{L^{2}(\omega)}^{2}+\|\nabla w\|_{L^{2}(\omega)}^{2}\right)^{1 / 2}
$$


Taking into account that

$$
\tilde{A}_{a}\left(x^{\prime}\right) \zeta \cdot \zeta \geq|\zeta|^{2}, \quad \text { for all } \zeta \in \mathbb{R}^{2} \text { and } x^{\prime} \in \omega
$$

and that

$$
2 a|\xi| \int_{\omega}\left|x^{\prime \perp} \cdot \nabla v w\right| d x^{\prime} \leq a^{2} \delta^{2} \int_{\omega}|\nabla v|^{2} d x^{\prime}+\xi^{2} \int_{\omega} w^{2} d x^{\prime}, \quad(v, w) \in \mathcal{H},
$$

where

$$
\delta=\max _{x^{\prime} \in \omega}\left|x^{\prime}\right|<\infty
$$

it is easy to see that

$$
\mathcal{A}_{\xi}[(v, w),(v, w)] \geq\left(1-a^{2} \delta^{2}\right)\|(v, w)\|_{\mathcal{H}}^{2} .
$$

Let us fix $a_{0}>0$ so small that $\alpha=1-a_{0}^{2} \delta^{2}>0$. In light of the above estimate, the bilinear form $\mathcal{A}_{\xi}$ is $\alpha$-elliptic for every $\xi \in \mathbb{R}$, provided we have $|a| \leq a_{0}$. For each $\Phi \in C\left(\mathbb{R} ; \mathcal{H}^{\prime}\right)$ and every $\xi \in \mathbb{R}$, there is thus a unique $(v(\xi), w(\xi)) \in \mathcal{H}$ satisfying

$$
\mathcal{A}_{\xi}[(v(\xi), w(\xi)),(\varphi, \psi)]=\langle\Phi(\xi),(\varphi, \psi)\rangle \quad \text { for all }(\varphi, \psi) \in \mathcal{H},
$$

by Lax-Milgram's lemma. From this then follows that

$$
\begin{aligned}
\mathcal{A}_{\xi+\eta} & {[(v(\xi+\eta)-v(\xi), w(\xi+\eta)-w(\xi)),(\varphi, \psi)] } \\
=\mathcal{A}_{\xi} & {[(v(\xi), w(\xi)),(\varphi, \psi)]-\mathcal{A}_{\xi+\eta}[(v(\xi), w(\xi)),(\varphi, \psi)] } \\
& +\langle\Phi(\xi+\eta)-\Phi(\xi),(\varphi, \psi)\rangle,
\end{aligned}
$$

for each $\xi, \eta \in \mathbb{R}$ and $(\varphi, \psi) \in \mathcal{H}$. Further, by observing through elementary computations that

$$
\begin{aligned}
\mathcal{A}_{\xi+\eta}[(v, w),(\varphi, \psi)]= & \mathcal{A}_{\xi+\eta}[(v, w),(\varphi, \psi)] \\
& -2 a \eta \int_{\omega} x^{\prime \perp} \cdot\left(\varphi \nabla_{x^{\prime}} w-\psi \nabla_{x^{\prime}} v\right) d x^{\prime} \\
& +\eta(2 \xi+\eta) \int_{\omega}(v \varphi+w \psi) d x^{\prime},
\end{aligned}
$$

for every $(v, w),(\varphi, \psi) \in \mathcal{H}$, we deduce from (4.11) and Poincaré's inequality that there exists a constant

$$
C=C\left(\xi, \omega, a_{0}\right)>0
$$


satisfying

$$
\begin{aligned}
& \mathcal{A}_{\xi}[(v(\xi), w(\xi)),(v(\xi+\eta)-v(\xi), w(\xi+\eta)-w(\xi))] \\
& \quad-\mathcal{A}_{\xi+\eta}[(v(\xi), w(\xi)),(v(\xi+\eta)-v(\xi), w(\xi+\eta)-w(\xi))] \\
& \quad \leq C|\eta|\|(v(\xi), w(\xi))\|_{\mathcal{H}}\|(v(\xi+\eta)-v(\xi), w(\xi+\eta)-w(\xi))\|_{\mathcal{H}},
\end{aligned}
$$

for all $\xi \in \mathbb{R}$ and $\eta \in[-1,1]$. In light of (4.9) and (4.11) written with

$$
(\varphi, \psi)=(v(\xi+\eta)-v(\xi), w(\xi+\eta)-w(\xi))
$$

and (4.12), we thus find out that

$$
\begin{aligned}
& \alpha\|(v(\xi+\eta)-v(\xi), w(\xi+\eta)-w(\xi))\|_{\mathcal{H}} \\
& \quad \leq C|\eta|\|(v(\xi), w(\xi))\|_{\mathcal{H}}+\|\Phi(\xi+\eta)-\Phi(\xi)\|_{\mathcal{H}^{\prime}} .
\end{aligned}
$$

This proves that $(v, w) \in C(\mathbb{R} ; \mathcal{H})$. Moreover, we obtain

$$
\|(v(\xi), w(\xi))\|_{\mathcal{H}} \leq(1 / \alpha)\|\Phi(\xi)\|_{\mathcal{H}^{\prime}}, \quad \xi \in \mathbb{R},
$$

directly from (4.9)-(4.10). Further, it is easy to check for $\Phi \in C^{1}\left(\mathbb{R} ; \mathcal{H}^{\prime}\right)$ that $\left(v^{\prime}(\xi), w^{\prime}(\xi)\right) \in C(\mathbb{R}, \mathcal{H})$ is the solution to the variational problem

$$
\begin{aligned}
& \mathcal{A}_{\xi}\left[\left(\left(v^{\prime}(\xi), w^{\prime}(\xi)\right),(\varphi, \psi)\right]\right. \\
& \quad=\left\langle\Phi_{0}(\xi),(\varphi, \psi)\right\rangle+\left\langle\Phi^{\prime}(\xi),(\varphi, \psi)\right\rangle \quad \text { for all }(\varphi, \psi) \in \mathcal{H},
\end{aligned}
$$

where

$$
\begin{aligned}
& \left\langle\Phi_{0}(\xi),(\varphi, \psi)\right\rangle \\
& \quad=2 a \int_{\omega} x^{\prime \perp} \cdot\left(\varphi \nabla_{x^{\prime}} w(\xi)-\psi \nabla_{x^{\prime}} v(\xi)\right) d x^{\prime}-2 \xi \int_{\omega}(v(\xi) \varphi+w(\xi) \psi) d x^{\prime} .
\end{aligned}
$$

Using (4.13) and noting $\langle\xi\rangle=\left(1+|\xi|^{2}\right)^{1 / 2}$, we deduce from the above estimate that

$$
\left\|\left(v^{\prime}(\xi), w^{\prime}(\xi)\right)\right\|_{\mathcal{H}} \leq C\left(\langle\xi\rangle\|\Phi(\xi)\|_{\mathcal{H}^{\prime}}+\left\|\Phi^{\prime}(\xi)\right\|_{\mathcal{H}^{\prime}}\right), \quad \xi \in \mathbb{R},
$$

for some constant $C=C\left(a_{0}, \omega\right)>0$. Similarly, if $\Phi \in C^{2}\left(\mathbb{R} ; \mathcal{H}^{\prime}\right)$ then the same reasoning shows that $(v, w) \in C^{2}(\mathbb{R}, \mathcal{H})$ verifies

$\left\|\left(v^{\prime \prime}(\xi), w^{\prime \prime}(\xi)\right)\right\|_{\mathcal{H}} \leq C\left(\langle\xi\rangle^{2}\|\Phi(\xi)\|_{\mathcal{H}^{\prime}}+\langle\xi\rangle\left\|\Phi^{\prime}(\xi)\right\|_{\mathcal{H}^{\prime}}+\left\|\Phi^{\prime \prime}(\xi)\right\|_{\mathcal{H}^{\prime}}\right), \quad \xi \in \mathbb{R}$.

Summing up we obtain the following result. 
Proposition 4.1. Let $a_{0} \in\left(0,\left(\max _{x^{\prime} \in \omega}\left|x^{\prime}\right|\right)^{-1 / 2}\right)$, pick $a \in\left[-a_{0}, a_{0}\right]$ and assume that $\alpha\left(x_{3}\right)=$ a for all $x_{3} \in \mathbb{R}$. Then for every $\Phi \in H^{2}\left(\mathbb{R}, \mathcal{H}^{\prime}\right)$ such that $\langle\xi\rangle^{2-j} \Phi^{(j)} \in L^{2}\left(\mathbb{R}, \mathcal{H}^{\prime}\right), j=0,1$, the variational problem (4.10) admits a unique solution $(v, w) \in H^{2}(\mathbb{R} ; \mathcal{H})$ satisfying

$$
\|(v, w)\|_{H^{2}(\mathbb{R} ; \mathcal{H})} \leq C\left(\sum_{j=0}^{2}\left\|\langle\xi\rangle^{2-j} \Phi^{(j)}\right\|_{L^{2}\left(\mathbb{R} ; \mathcal{H}^{\prime}\right)}\right),
$$

for some constant $C=C\left(\omega, a_{0}\right)>0$. The above assumptions on $\Phi$ are actually satisfied whenever $\Phi=\widehat{\Psi}$ for some $\Psi \in H^{2}\left(\mathbb{R} ; \mathcal{H}^{\prime}\right)$ such that $x_{3} \Psi \in H^{1}\left(\mathbb{R} ; \mathcal{H}^{\prime}\right)$ and $x_{3}^{2} \Psi \in L^{2}\left(\mathbb{R} ; \mathcal{H}^{\prime}\right)$. Moreover, the estimate (4.14) reads

$$
\|(v, w)\|_{H^{2}(\mathbb{R} ; \mathcal{H})} \leq C\left(\sum_{j=0}^{2}\left\|x_{3}^{j} \Psi\right\|_{H^{2-j}\left(\mathbb{R} ; \mathcal{H}^{\prime}\right)}\right),
$$

in this case.

Armed with Proposition 4.1 we may now tackle the analysis of the solution of equation (4.8).

Some useful properties of the solution to (4.8). Pick $F \in H^{1}(\omega)$ such that $F=f$ on $\partial \omega$ and $\|F\|_{H^{1}(\omega)} \leq C(\omega)\|f\|_{H^{1 / 2}(\partial \omega)}$. Let $\tilde{u}^{r}$ (resp., $\tilde{u}^{i}$ ) denote the real (resp., imaginary) part of $\tilde{u}=\hat{u}-\hat{g}(\xi) F=\tilde{u}^{r}+i \tilde{u}^{i}$. Since the Fourier transform $\hat{u}$ of the $H^{1}(\Omega)$-solution $u$ to (1.3) is actually solution to (4.8), we get by direct calculation that $\left(\tilde{u}^{r}, \tilde{u}^{i}\right)$ is solution to the variational problem (4.10), with

$$
\langle\Phi(\xi),(\varphi, \psi)\rangle=-\mathcal{A}_{\xi}\left[\left(\hat{g}^{r} F, \hat{g}^{i} F\right),(\varphi, \psi)\right],
$$

and where $\hat{g}^{r}$ (resp., $\hat{g}^{i}$ ) stands for the real (resp., imaginary) part of $\hat{g}$. In light of (4.16) we check out using elementary computations that

$$
\begin{aligned}
\|\Phi(\xi)\|_{\mathcal{H}^{\prime}} & \leq C\langle\xi\rangle^{2}|\hat{g}(\xi)|\|f\|_{H^{1 / 2}(\partial \omega)}, \\
\left\|\Phi^{\prime}(\xi)\right\|_{\mathcal{H}^{\prime}} & \leq C\left(\langle\xi\rangle^{2}\left|\hat{g}^{\prime}(\xi)\right|+\langle\xi\rangle|\hat{g}(\xi)|\right) \mid\|f\|_{H^{1 / 2}(\partial \omega)}, \\
\left\|\Phi^{\prime \prime}(\xi)\right\|_{\mathcal{H}^{\prime}} & \leq C\left(\langle\xi\rangle^{2}\left|\hat{g}^{\prime \prime}(\xi)\right|+\langle\xi\rangle\left|\hat{g}^{\prime}(\xi)\right|+|\hat{g}(\xi)|\right)\|f\|_{H^{1 / 2}(\partial \omega)},
\end{aligned}
$$

for some constant $C=C\left(a_{0}, \omega\right)>0$. Therefore we have $\langle\xi\rangle^{j} \Phi^{(2-j)} \in L^{2}(\mathbb{R})$ for $j=0,1,2$, provided $\langle\xi\rangle^{4-j} \hat{g}^{j} \in L^{2}(\mathbb{R})$, which is actually the case if $x_{3}^{j} g \in$ $H^{4-j}(\mathbb{R})$. From this and Proposition 4.1 then follows the following corollary. 
Corollary 4.1. Let $a$ and $\alpha$ be the same as in Proposition 4.1 and assume that $g \in H^{4}(\mathbb{R})$ verifies

$$
x_{3} g \in H^{3}(\mathbb{R}), \quad x_{3}^{2} g \in H^{2}(\mathbb{R}),
$$

and

$$
\int_{\mathbb{R}} g\left(x_{3}\right) d x_{3}=1
$$

Then we have

$$
\hat{u} \in H^{2}\left(\mathbb{R} ; H^{1}(\omega)\right), \quad u \in L^{1}\left(\mathbb{R} ; H^{1}(\omega)\right),
$$

and

$$
U=\hat{u}(\cdot, 0)=\int_{\mathbb{R}} u\left(\cdot, x_{3}\right) d x_{3} \in H^{1}(\omega)
$$

is the variational solution to the BVP

$$
\begin{cases}\operatorname{div}_{x^{\prime}}\left(\widetilde{A_{a}} \nabla_{x^{\prime}} U\right)=0 & \text { in } \omega, \\ U=f & \text { on } \partial \omega .\end{cases}
$$

In view of (4.8) and (4.10), we deduce from (4.17)-(4.19) that

$$
\begin{gathered}
\left\|\operatorname{div}_{x^{\prime}}\left(\tilde{A_{a}} \nabla_{x^{\prime}} \hat{u}(\cdot, \xi)\right)\right\|_{L^{2}(\omega)} \leq C\langle\xi\rangle^{4}|\hat{g}(\xi)|\|f\|_{H^{1 / 2}(\partial \omega)}, \\
\left\|\partial_{\xi} \operatorname{div}_{x^{\prime}}\left(\widetilde{A_{a}} \nabla_{x^{\prime}} \hat{u}(\cdot, \xi)\right)\right\|_{L^{2}(\omega)} \leq C\left(\langle\xi\rangle^{5}\left|\hat{g}^{\prime}(\xi)\right|+\langle\xi\rangle^{4}|\hat{g}(\xi)|\right) \mid\|f\|_{H^{1 / 2}(\partial \omega)},
\end{gathered}
$$

and

$$
\begin{aligned}
& \left\|\partial_{\xi}^{2} \operatorname{div}_{x^{\prime}}\left(\tilde{A}_{a} \nabla_{x^{\prime}} \hat{u}(\cdot, \xi)\right)\right\|_{L^{2}(\omega)} \\
& \quad \leq C\left(\langle\xi\rangle^{6}|\hat{g}(\xi)|+\langle\xi\rangle^{5}\left|\hat{g}^{\prime}(\xi)\right|+\langle\xi\rangle^{4}\left|\hat{g}^{\prime \prime}(\xi)\right|\right)\|f\|_{H^{1 / 2}(\partial \omega)},
\end{aligned}
$$

for some positive constant $C$ depending only on $a_{0}$ and $\omega$. This combined with Corollary 4.1 yields the following result.

Proposition 4.2. Let $a$ and $\alpha$ be as in Proposition 4.1. Let $g \in H^{6}(\mathbb{R})$ verify

$$
x_{3} g \in H^{5}(\mathbb{R}), \quad x_{3}^{2} g \in H^{4}(\mathbb{R}),
$$

and

$$
\int_{\mathbb{R}} g\left(x_{3}\right) d x_{3}=1 \text {. }
$$


Then we have

$$
\widetilde{A}_{a} \nabla_{x^{\prime}} \hat{u} \cdot v\left(x^{\prime}\right) \in H^{2}\left(\mathbb{R} ; H^{-1 / 2}(\partial \omega)\right),
$$

and thus

$$
A_{a} \nabla u \cdot v(x) \in L^{1}\left(\mathbb{R} ; H^{-1 / 2}(\partial \omega)\right),
$$

with

$$
\begin{aligned}
\tilde{A}_{a} \nabla_{x^{\prime}} U \cdot v\left(x^{\prime}\right) & =\tilde{A}_{a} \nabla_{x^{\prime}} \hat{u}(\cdot, 0) \cdot v\left(x^{\prime}\right) \\
& =\int_{\mathbb{R}} A_{a} \nabla u\left(\cdot, x_{3}\right) \cdot v\left(x^{\prime}\right) d x_{3} \in H^{-1 / 2}(\partial \omega) .
\end{aligned}
$$

In light of Proposition 4.2 it is natural to define the two DN maps

$$
\Lambda_{a}: f \in H^{1 / 2}(\partial \omega) \longmapsto\left[x_{3} \longmapsto A_{a} \nabla u\left(\cdot, x_{3}\right) \cdot v\left(\cdot, x_{3}\right)\right] \in L^{1}\left(\mathbb{R} ; H^{-1 / 2}(\partial \omega)\right)
$$

and

$$
\tilde{\Lambda}_{a}: f \in H^{1 / 2}(\partial \omega) \longmapsto \widetilde{A}_{a} \nabla_{x^{\prime}} U \cdot v\left(x^{\prime}\right) \in H^{-1 / 2}(\partial \omega) .
$$

These two operators are bounded, and they satisfy the estimate

$$
\begin{aligned}
& \left\|\tilde{\Lambda}_{1}-\tilde{\Lambda}_{2}\right\|_{\mathcal{L}\left(H^{1 / 2}(\partial \omega), H^{-1 / 2}(\partial \omega)\right)} \\
& \quad \leq\left\|\Lambda_{1}-\Lambda_{2}\right\|_{\mathcal{L}\left(H^{1 / 2}(\partial \omega), L^{1}\left(\mathbb{R} ; H^{-1 / 2}(\partial \omega)\right)\right)},
\end{aligned}
$$

where, for simplicity, we write $\Lambda_{j}$ (resp., $\left.\widetilde{\Lambda}_{j}\right)$ for $\Lambda_{a_{j}}\left(\right.$ resp., $\left.\widetilde{\Lambda}_{a_{j}}\right), j=1,2$.

Finally, since the matrix $\partial_{a} \widetilde{A}\left(x^{\prime}, a\right)$ has two eigenvalues $\lambda_{0}=0$ and $\lambda_{1}=\left|x^{\prime}\right|^{2}$, we derive the following result by mimicking the proof of [2, Claim, page 169].

Theorem 4.1. Let $a_{0}$ be the same as in Proposition 4.1, let $a_{j} \in \mathbb{R}, j=1,2$, and assume that $\alpha_{j}\left(x_{3}\right)=a_{j}$ for all $x_{3} \in \mathbb{R}$. Then there exists a constant $C>0$, depending only on $a_{0}$ and $\omega$, such that the stability estimate

$$
\left|a_{1}-a_{2}\right| \leq C\left\|\Lambda_{1}-\Lambda_{2}\right\|_{\mathcal{L}\left(H^{1 / 2}(\partial \omega), L^{1}\left(\mathbb{R} ; H^{-1 / 2}(\partial \omega)\right)\right)},
$$

holds true whenever $\left|a_{1}\right|,\left|a_{2}\right| \leq a_{0}$. 


\section{Appendixes}

\section{A. Restriction to $\tilde{H}^{3 / 2}(\partial \Omega)$}

In this subsection we exhibit sufficient conditions on $\omega$ and $\alpha$ ensuring that the restriction of $\Lambda_{\alpha}$ to $\widetilde{H}^{3 / 2}(\partial \Omega)$ is a bounded operator into $L^{2}\left(\mathbb{R} ; H^{1 / 2}(\partial \omega)\right)$. We assume for this purpose that $\Omega_{1}=\omega \times(-1,1)$ has $H^{2}$-regularity property. That is, for every $F \in L^{2}(\Omega)$ and any matrix-valued function $C=\left(C_{i j}(x)\right)_{1 \leq i, j \leq 3}$ with coefficients in $W^{1, \infty}\left(\Omega_{1}\right)$ verifying the ellipticity condition

there exists $\alpha>0$, such that, for all $\xi \in \mathbb{R}^{3}, x \in \Omega_{1}, \quad C(x) \xi \cdot \xi \geq \alpha|\xi|^{2}$, the BVP

$$
\begin{cases}\operatorname{div}(C \nabla w)=F & \text { in } \Omega_{1}, \\ w=0 & \text { on } \partial \Omega_{1},\end{cases}
$$

has a unique solution $w \in H^{2}\left(\Omega_{1}\right)$ obeying

$$
\|w\|_{H^{2}\left(\Omega_{1}\right)} \leq C(\alpha, M)\|F\|_{L^{2}\left(\Omega_{1}\right)},
$$

for some constant $C(\alpha, M)>0$ depending only on $\alpha$,

$$
M=\max _{1 \leq i, j \leq 3}\left\|C_{i j}\right\|_{W^{1, \infty}\left(\Omega_{1}\right)}
$$

and $\omega$.

Note that $\Omega_{1}$ has $H^{2}$-regularity property if and only if this is the case for $\Omega_{a}=\omega \times(-a, a)$ and some $a>0$. Moreover we recall from [14] that $\Omega_{1}$ has $H^{2}$-regularity property provided $\omega$ is convex.

We turn now to establishing the following result, which is our main tool for the analysis of the restriction of $\Lambda_{\alpha}$ to $\tilde{H}^{3 / 2}(\partial \Omega)$.

Theorem A.1. Assume that $\alpha \in C^{0,1}(\mathbb{R})$ and that $\Omega_{1}$ has $H^{2}$-regularity property. Then for any $f \in \widetilde{H}^{3 / 2}(\partial \Omega)$, the $B V P(1.3)$ admits a unique solution $u \in H^{2}(\Omega)$. Moreover there is a constant $C>0$, depending only on $\|\alpha\|_{C^{0,1}(\mathbb{R})}$ and $\omega$, such that we have

$$
\|u\|_{H^{2}(\Omega)} \leq C\|f\|_{\widetilde{H}^{3 / 2}(\partial \Omega)} .
$$


Proof. Since $f \in \widetilde{H}^{3 / 2}(\partial \Omega)$ we may choose $F \in H^{2}(\Omega)$ in accordance with Lemma 2.1 so that $F=f$ on $\partial \Omega$ and

$$
\|F\|_{H^{2}(\Omega)}=\|f\|_{\widetilde{H}^{3 / 2}(\partial \Omega)} .
$$

We put $\Psi=\operatorname{div}(A \nabla F)$. By the ellipticity condition (2.3) we have a unique $u_{0} \in$ $H_{0}^{1}(\Omega)$ satisfying simultaneously

$$
\int_{\Omega} A \nabla u_{0} \cdot \nabla v d x=\int_{\Omega} \Psi v d x, \text { for all } v \in H_{0}^{1}(\Omega),
$$

and

$$
\left\|u_{0}\right\|_{H^{1}(\Omega)} \leq C_{0}\|\Psi\|_{L^{2}(\Omega)},
$$

for some constant $C_{0}>0$ depending on $\omega$ and $M=\|\alpha\|_{C^{0,1}(\mathbb{R})}$.

Further, there exists $\xi_{n} \in C_{0}^{\infty}(-(n+1), n+1)$ such that $\xi_{n}=1$ in $[-n, n]$, $\left\|\xi_{n}^{\prime}\right\|_{\infty} \leq \kappa$ and $\left\|\xi_{n}^{\prime \prime}\right\|_{\infty} \leq \kappa$ for all $n \geq 1$, by [17, Theorem 1.4.1 and formula (1.4.2)] and [17, p. 25], where $\kappa$ is a constant which is independent of $n$.

Thus, we get for any $v \in H_{0}^{1}(\Omega)$ and $n \geq 1$ that

$$
\begin{aligned}
\int_{\Omega} A \nabla\left(\xi_{n} u_{0}\right) \cdot \nabla v d x \\
\quad=\int_{\Omega} A \nabla u_{0} \cdot \nabla\left(\xi_{n} v\right) d x-\int_{\Omega}\left(A \nabla u_{0} \cdot \nabla \xi_{n}\right) v d x+\int_{\Omega}\left(A \nabla \xi_{n} \cdot \nabla v\right) u_{0} d x,
\end{aligned}
$$

by direct calculation. An integration by parts in the last term of this identity providing

$$
\int_{\Omega}\left(A \nabla \xi_{n} \cdot \nabla v\right) u_{0} d x=-\int_{\Omega}\left(A \nabla \xi_{n} \cdot \nabla u_{0}\right) v d x-\int_{\Omega} \operatorname{div}\left(A \nabla \xi_{n}\right) u_{0} v d x,
$$

we find out that

$$
\begin{aligned}
\int_{\Omega} A \nabla\left(\xi_{n} u_{0}\right) \cdot \nabla v d x= & \int_{\Omega} A \nabla u_{0} \cdot \nabla\left(\xi_{n} v\right) d x-\int_{\Omega}\left(A \nabla u_{0} \cdot \nabla \xi_{n}\right) v d x \\
& -\int_{\Omega}\left(A \nabla \xi_{n} \cdot \nabla u_{0}\right) v d x-\int_{\Omega} \operatorname{div}\left(A \nabla \xi_{n}\right) u_{0} v d x .
\end{aligned}
$$

Since $A$ is symmetric, it follows from this and (A.3) that

$$
\begin{aligned}
\int_{\Omega} A \nabla\left(\xi_{n} u_{0}\right) \cdot \nabla v d x=\int_{\Omega} & \Psi \xi_{n} v d x-2 \int_{\Omega}\left(A \nabla \xi_{n} \cdot \nabla u_{0}\right) v d x \\
& \quad-\int_{\Omega} \operatorname{div}\left(A \nabla \xi_{n}\right) u_{0} v d x, \quad \text { for all } v \in H_{0}^{1}(\Omega) .
\end{aligned}
$$


Therefore, bearing in mind that $\Omega_{a}=\omega \times(-a, a)$ for any $a>0$, the $\xi_{n} u_{0} \in$ $H_{0}^{1}\left(\Omega_{n+1}\right)$ is thus solution to the variational problem

$$
\int_{\Omega_{n+1}} A \nabla\left(\xi_{n} u_{0}\right) \cdot \nabla v d x=\int_{\Omega_{n+1}} \tilde{\Psi} v d x, \quad \text { for all } v \in H_{0}^{1}\left(\Omega_{n+1}\right),
$$

with

$$
\widetilde{\Psi}=\Psi \xi_{n}-2 A \nabla \xi_{n} \cdot \nabla u_{0}-\operatorname{div}\left(A \nabla \xi_{n}\right) u_{0} .
$$

The next step of the proof is to make the change of variables

$$
\left(x^{\prime}, x_{3}\right) \in \Omega_{n+1} \longmapsto\left(x^{\prime}, y_{3}\right)=\left(x^{\prime}, 1 /(n+1) x_{3}\right) \in \Omega_{1}
$$

in (A.5). Putting

$$
J_{n}=\left(\begin{array}{ccc}
1 & 0 & 0 \\
0 & 1 & 0 \\
0 & 0 & 1 / n
\end{array}\right), \quad n \geq 1
$$

and

$$
\begin{aligned}
\underline{A}\left(x^{\prime}, y_{3}\right) & =1 /(n+1) J_{n+1} A\left(x^{\prime},(n+1) y_{3}\right) J_{n+1}, \\
\underline{\xi}\left(y_{3}\right) & =\xi_{n}\left((n+1) y_{3}\right), \\
\underline{u}\left(x^{\prime}, y_{3}\right) & =u_{0}\left(x^{\prime},(n+1) y_{3}\right), \\
w_{n}\left(x^{\prime}, y_{3}\right)= & \xi_{n}\left((n+1) y_{3}\right) u_{0}\left(x^{\prime},(n+1) y_{3}\right), \\
\underline{\operatorname{div}}\left(P\left(x^{\prime}, y_{3}\right)\right)= & \partial_{x_{1}} P_{1}\left(x^{\prime}, y_{3}\right)+\partial_{x_{2}} P_{2}\left(x^{\prime}, y_{3}\right) \\
& +1 /(n+1) \partial_{y_{3}} P_{3}\left(x^{\prime}, y_{3}\right), \\
\underline{\Psi}\left(x^{\prime}, y_{3}\right)= & 1 /(n+1)\left[\Psi\left(x^{\prime},(n+1) y_{3}\right)\right. \\
& \quad-2 J_{n+1} A\left(x^{\prime},(n+1) y_{3}\right) J_{n+1} \nabla \underline{\xi}\left(y_{3}\right) \cdot \nabla \underline{u}\left(x^{\prime}, y_{3}\right) \\
& \left.\quad \underline{\operatorname{div}}\left(A\left(x^{\prime},(n+1) y_{3}\right) J_{n+1} \nabla \underline{\xi}\left(y_{3}\right)\right) \underline{u}\left(x^{\prime}, y_{3}\right)\right],
\end{aligned}
$$

for $\left(x^{\prime}, y_{3}\right) \in \Omega_{1}$, we find out by direct computations that $w_{n} \in H_{0}^{1}\left(\Omega_{1}\right)$ is solution to

$$
\int_{\Omega_{1}} \underline{A} \nabla w_{n} \cdot \nabla v d x=\int_{\Omega_{1}} \underline{\Psi} v d x, \text { for all } v \in H_{0}^{1}\left(\Omega_{1}\right)
$$


Since

$$
\|\underline{\Psi}\|_{L^{2}\left(\Omega_{1}\right)} \leq(n+1)^{-3 / 2} C(\omega, M)\|\Psi\|_{L^{2}(\Omega)}
$$

by (A.4), where

$$
C=C(\omega, M)
$$

denotes some generic positive constant depending only on $\omega$ and $M$, it holds true that

$$
\left\|w_{n}\right\|_{H^{2}\left(\Omega_{1}\right)} \leq(n+1)^{-3 / 2} C(\omega, M)\|\Psi\|_{L^{2}(\Omega)} .
$$

Here we used the estimate

$$
\left\|w_{n}\right\|_{H^{2}\left(\Omega_{1}\right)} \leq C(\omega, M)\|\underline{\Psi}\|_{L^{2}\left(\Omega_{1}\right)},
$$

arising from the $H^{2}$-regularity property imposed on $\Omega_{1}$. As a consequence, we have

$$
\left\|\xi_{n} u_{0}\right\|_{H^{2}(\Omega)} \leq C(\omega, M)\|\Psi\|_{L^{2}(\Omega)} .
$$

Therefore, upon eventually extracting a subsequence of $\left(\xi_{n} u_{0}\right)_{n}$, we may assume that it converges weakly to $\tilde{u}$ in $H^{2}(\Omega)$. On the other hand $\left(\xi_{n} u_{0}\right)_{n}$ converges to $u_{0}$ in $L^{2}(\Omega)$. Thus by the uniqueness of the limit, we have $u_{0}=\tilde{u} \in H^{2}(\Omega)$ so $\left(\xi_{n} u_{0}\right)_{n}$ converges weakly to $u_{0}$ in $H^{2}(\Omega)$. Further, the norm $\|\cdot\|_{H^{2}(\Omega)}$ being lower semi-continuous, we have

$$
\left\|u_{0}\right\|_{H^{2}(\Omega)} \leq \liminf _{n}\left\|\xi_{n} u_{0}\right\|_{H^{2}(\Omega)} \leq C(\omega, M)\|\Psi\|_{L^{2}(\Omega)},
$$

by (A.6). Bearing in mind that $\|\Psi\|_{L^{2}(\Omega)} \leq C(\omega, M)\|F\|_{H^{2}(\Omega)}$, (A.2) and (A.7) then yield

$$
\left\|u_{0}\right\|_{H^{2}(\Omega)} \leq C(\omega, M)\|f\|_{\widetilde{H}^{3 / 2}(\partial \Omega)} .
$$

Now the desired result follows from this by taking into account that $u=u_{0}+F \in$ $H^{2}(\Omega)$ is the unique solution to (1.3).

For all $\Omega$ and $\alpha$ fulfilling the assumptions of Theorem A.1, the mapping

$$
\Lambda_{\alpha}: f \in \widetilde{H}^{3 / 2}(\partial \Omega) \longmapsto \partial_{\nu} u \in L^{2}\left(\mathbb{R} ; H^{1 / 2}(\partial \omega)\right),
$$

where $u$ denotes the unique $H^{2}(\Omega)$-solution to (1.3), is well defined by Theorem A.1. Further, since $C_{0}^{\infty}\left(\mathbb{R} ; H^{2}(\omega)\right)$ is dense in $H^{2}(\Omega)$, then the trace operator

$$
\tilde{\tau}: w \in H^{2}(\Omega) \longmapsto \partial_{\nu} w \in L^{2}\left(\mathbb{R} ; H^{1 / 2}(\partial \omega)\right),
$$

is easily seen to be bounded. From this and (A.1) then follows that $\left\|\Lambda_{\alpha}\right\| \leq C$ as a linear bounded operator from $\widetilde{H}^{3 / 2}(\partial \Omega)$ into $L^{2}\left(\mathbb{R} ; H^{1 / 2}(\partial \omega)\right)$, where the constant $C>0$ depends only on $\omega$ and $\|\alpha\|_{C^{0,1}(\mathbb{R})}$. 


\section{B. Linking $\Lambda_{\alpha}$ to the DN map associated with $\Omega_{\theta}$}

In this subsection we define the DN map $\tilde{\Lambda}_{\theta}$ associated with the BVP (1.2), which is stated on the twisted domain $\Omega_{\theta}$, and establish the link between $\tilde{\Lambda}_{\theta}$ and $\Lambda_{\alpha}$, where we recall that $\alpha=\theta^{\prime}$. It turns out that $\widetilde{\Lambda}_{\theta}$ is not physically relevant ${ }^{4}$ for the analysis of the inverse problem under consideration in this text, but since the BVP (1.3) was derived from (1.2), it is plainly natural to link the operator $\Lambda_{\alpha}$ we used in the preceding sections to $\widetilde{\Lambda}_{\theta}$.

We start by defining the trace space for functions in $H^{1}\left(\Omega_{\theta}\right)$. We set for all $L>0$,

$$
\begin{aligned}
\Omega_{\theta}^{L} & =\left\{\left(R_{\theta\left(x_{3}\right)} x^{\prime}, x_{3}\right) ; x^{\prime}=\left(x_{1}, x_{2}\right) \in \omega, x_{3} \in(-L, L)\right\} \\
& =\left\{x \in \Omega_{\theta},\left|x_{3}\right|<L\right\}
\end{aligned}
$$

and

$$
\begin{aligned}
\Gamma_{\theta}^{L} & =\left\{\left(R_{\theta\left(x_{3}\right)} x^{\prime}, x_{3}\right) ; x^{\prime}=\left(x_{1}, x_{2}\right) \in \partial \omega, x_{3} \in[-L, L]\right\} \\
& =\left\{x \in \partial \Omega_{\theta},\left|x_{3}\right|<L\right\} .
\end{aligned}
$$

For every $u \in H^{1}\left(\Omega_{\theta}\right)$ we have $u_{\mid \Omega_{\theta}^{L}} \in H^{1}\left(\Omega_{\theta}^{L}\right)$ hence $u_{\mid \partial \Omega_{\theta}^{L}} \in H^{1 / 2}\left(\partial \Omega_{\theta}^{L}\right)$. Thus, putting

$$
H^{1 / 2}\left(\Gamma_{\theta}^{L}\right)=\left\{h=g_{\mid \Gamma_{\theta}^{L}} \text { in } L^{2}\left(\Gamma_{\theta}^{L}\right) ; g \in H^{1 / 2}\left(\partial \Omega_{\theta}^{L}\right)\right\},
$$

it holds true that $u_{\mid \Gamma_{\theta}^{L}} \in H^{1 / 2}\left(\Gamma_{\theta}^{L}\right)$. Here the space $H^{1 / 2}\left(\Gamma_{\theta}^{L}\right)$ is equipped with its natural quotient norm

$$
\|h\|_{H^{1 / 2}\left(\Gamma_{\theta}^{L}\right)}=\inf \left\{\|g\|_{H^{1 / 2}\left(\partial \Omega_{\theta}^{L}\right)} ; g_{\mid \Gamma_{\theta}^{L}}=h\right\} .
$$

Further we define

$$
H_{\mathrm{loc}}^{1 / 2}\left(\partial \Omega_{\theta}\right)=\left\{h \in L_{\mathrm{loc}}^{2}\left(\partial \Omega_{\theta}\right) ; h_{\mid \Gamma_{\theta}^{L}} \in H^{1 / 2}\left(\Gamma_{\theta}^{L}\right) \text { for all } L>0\right\},
$$

and then introduce the subspace

$$
\begin{array}{r}
\widetilde{H}^{1 / 2}\left(\partial \Omega_{\theta}\right)=\left\{h \in H_{\mathrm{loc}}^{1 / 2}\left(\partial \Omega_{\theta}\right) ; \text { there exists } v \in H^{1}\left(\Omega_{\theta}\right)\right. \\
\text { such that } \left.v_{\mid \partial \Omega_{\theta}}=h\right\}
\end{array}
$$

of $H_{\mathrm{loc}}^{1 / 2}\left(\partial \Omega_{\theta}\right)$.

${ }^{4}$ Since $\widetilde{\Lambda}_{\theta}$ is defined from the Neumann observation on $\partial \Omega_{\theta}$ of the solution to (1.2) then the variable twisting angle $\theta \in C^{1}(\mathbb{R})$ should necessarily be known everywhere. 
Here and henceforth $v_{\mid \partial \Omega_{\theta}}=h$ means that the identity

$$
v_{\mid \Gamma_{\theta}^{L}}=h_{\mid \Gamma_{\theta}^{L}}
$$

holds in the trace sense for every $L>0$. It is not hard to see that $\widetilde{H}^{1 / 2}\left(\partial \Omega_{\theta}\right)$ is a Banach space for the quotient norm

$$
\|h\|_{\widetilde{H}^{1 / 2}\left(\partial \Omega_{\theta}\right)}=\inf \left\{\|v\|_{H^{1}\left(\Omega_{\theta}\right)} ; v_{\mid \partial \Omega_{\theta}}=h\right\} .
$$

We introduce the mapping

$$
\begin{aligned}
I_{\theta}: C_{0}^{1}\left(\partial \Omega_{\theta}\right) & \longrightarrow C_{0}^{1}(\partial \Omega), \\
g & \longmapsto f=g \circ \varphi_{\theta},
\end{aligned}
$$

where, for the sake of shortness, we note

$$
\varphi_{\theta}(x)=T_{\theta\left(x_{3}\right)}\left(x^{\prime}, x_{3}\right), \quad \text { for } x \in \bar{\Omega} .
$$

Pick $g$ in $C_{0}^{1}\left(\partial \Omega_{\theta}\right)$ and choose $v \in C_{0}^{1}\left(\mathbb{R}^{3}\right)$ such that $v_{\mid \partial \Omega_{\theta}}=g$. Since $v_{\mid \Omega_{\theta}} \in$ $H^{1}\left(\Omega_{\theta}\right)$ we get that $g \in \tilde{H}^{1 / 2}\left(\partial \Omega_{\theta}\right)$. Moreover for all $v \in H^{1}\left(\Omega_{\theta}\right)$ obeying $v_{\mid \partial \Omega_{\theta}}=g$ the function $u=v_{\mid \Omega_{\theta}} \circ \varphi_{\theta}$ belongs to $H^{1}(\Omega)$ by [5, Proposition 9.6], and

$$
\left\|I_{\theta} g\right\|_{\widetilde{H}^{1 / 2}(\partial \Omega)} \leq\|u\|_{H^{1}(\Omega)} \leq C(\omega, \theta)\|v\|_{H^{1}\left(\Omega_{\theta}\right)} .
$$

As a consequence, we have

$$
\left\|I_{\theta} g\right\|_{\widetilde{H}^{1 / 2}(\partial \Omega)} \leq C(\omega, \theta)\|g\|_{\widetilde{H}^{1 / 2}\left(\partial \Omega_{\theta}\right)}, \quad \text { for any } g \in C_{0}^{1}\left(\partial \Omega_{\theta}\right) .
$$

Let us now consider $g \in \widetilde{H}^{1 / 2}\left(\partial \Omega_{\theta}\right)$ and $v \in H^{1}\left(\Omega_{\theta}\right)$ such that

$$
v_{\mid \partial \Omega_{\theta}}=g .
$$

For any sequence $\left(v_{n}\right)_{n} \in C_{0}^{1}\left(\mathbb{R}^{3}\right)$ such that

$$
v_{n \mid \Omega_{\theta}} \longmapsto v \quad \text { in } H^{1}\left(\Omega_{\theta}\right) \text { as } n \rightarrow+\infty,
$$

it is clear that

$$
\left\|g-g_{n}\right\|_{\widetilde{H}^{1 / 2}\left(\partial \Omega_{\theta}\right)} \leq\left\|v-v_{n}\right\|_{H^{1}\left(\Omega_{\theta}\right)},
$$

provided $g_{n}=v_{n \mid \partial \Omega_{\theta}}$. Hence $\left(g_{n}\right)_{n}$ converges to $g$ in $\tilde{H}^{1 / 2}\left(\partial \Omega_{\theta}\right)$. 
For all $n \geq 1$, put

$$
f_{n}=I_{\theta} g_{n}=g_{n} \circ \varphi_{\theta} \quad \text { and } \quad u_{n}=v_{n} \circ \varphi_{\theta} .
$$

Since $f_{n}=u_{n \mid \partial \Omega}$, we see that

$$
\left\|f_{n}-f_{m}\right\|_{\widetilde{H}^{1 / 2}(\partial \Omega)} \leq\left\|u_{n}-u_{m}\right\|_{H^{1}(\Omega)} \leq C(\omega, \theta)\left\|v_{n}-v_{m}\right\|_{H^{1}\left(\Omega_{\theta}\right)} .
$$

Therefore $\left(f_{n}\right)_{n}$ is a Cauchy sequence in $\widetilde{H}^{1 / 2}(\partial \Omega)$ and $f=\lim _{n} f_{n} \in \widetilde{H}^{1 / 2}(\partial \Omega)$. Set $f=I_{\theta} g$. Then, in view of (B.1), $I_{\theta}$ extends to a bounded operator, still denoted by $I_{\theta}$, from $\widetilde{H}^{1 / 2}\left(\partial \Omega_{\theta}\right)$ into $\widetilde{H}^{1 / 2}(\partial \Omega)$.

Arguing as above, we thus find out that the mapping

$$
\begin{aligned}
J_{\theta}: C_{0}^{1}(\partial \Omega) & \longrightarrow C_{0}^{1}\left(\partial \Omega_{\theta}\right), \\
f & \longmapsto g=f \circ \psi_{\theta},
\end{aligned}
$$

where $\psi_{\theta}=\varphi_{\theta}^{-1}$, extends to a bounded operator, which is still called $J_{\theta}$, from $\widetilde{H}^{1 / 2}(\partial \Omega)$ into $\widetilde{H}^{1 / 2}\left(\partial \Omega_{\theta}\right)$.

Evidently,

$$
I_{\theta} J_{\theta} f=f, \quad \text { for all } f \in C_{0}^{1}(\partial \Omega),
$$

and

$$
J_{\theta} I_{\theta} g=g, \text { for all } g \in C_{0}^{1}\left(\partial \Omega_{\theta}\right) .
$$

Therefore we have

$$
J_{\theta}=I_{\theta}^{-1}
$$

from the density of $C_{0}^{1}(\partial \Omega)$ (resp., $\left.C_{0}^{1}\left(\partial \Omega_{\theta}\right)\right)$ in $\tilde{H}^{1 / 2}(\partial \Omega)\left(\right.$ resp., $\tilde{H}^{1 / 2}\left(\partial \Omega_{\theta}\right)$ ).

Next, by reasoning in the same way as in the derivation of (1.3), we prove with the help of the Lax-Milgram lemma that the BVP (1.2) has a unique solution $v \in H^{1}\left(\Omega_{\theta}\right)$ for every $g \in \widetilde{H}^{1 / 2}\left(\partial \Omega_{\theta}\right)$. Moreover the operator

$$
\tilde{\Lambda}_{\theta}: g \longmapsto \partial_{\nu} v
$$

is well defined as a bounded operator from $\widetilde{H}^{1 / 2}\left(\partial \Omega_{\theta}\right)$ into its dual space $\tilde{H}^{-1 / 2}\left(\partial \Omega_{\theta}\right)$. Similarly to $\Lambda_{\theta}$, it can be checked that $\tilde{\Lambda}_{\theta}$ is characterized by the following identity

$$
\left\langle\tilde{\Lambda}_{\theta} g, h\right\rangle=\int_{\Omega_{\theta}} \nabla v \cdot \nabla H d y,
$$

which holds true for all $h \in \widetilde{H}^{1 / 2}\left(\partial \Omega_{\theta}\right)$ and all $H \in H^{1}\left(\Omega_{\theta}\right)$ such that $H_{\mid \partial \Omega_{\theta}}=h$. By performing the change of variable $y=\varphi_{\theta}(x)$ in the last integral, we thus get that

$$
\left\langle\tilde{\Lambda}_{\theta} g, h\right\rangle=\int_{\Omega} A \nabla u \cdot \nabla\left(H \circ \varphi_{\theta}\right) d x,
$$


where $u$ denotes the solution to the BVP (1.3) with $f=I_{\theta} g$. Therefore we have

$$
\left\langle\tilde{\Lambda}_{\theta} g, h\right\rangle=\left\langle\Lambda_{\theta^{\prime}} I_{\theta} g, I_{\theta} h\right\rangle,
$$

which means that $\tilde{\Lambda}_{\theta}=I_{\theta}^{*} \Lambda_{\theta^{\prime}} I_{\theta}$, or equivalently that $\Lambda_{\theta^{\prime}}=J_{\theta}^{*} \widetilde{\Lambda}_{\theta} J_{\theta}$, where $\Lambda_{\theta^{\prime}}$ stands for $\Lambda_{\alpha}$.

\section{References}

[1] G. Alessandrini, Singular solutions of elliptic equations and the determination of conductivity by boundary measurements. J. Differential Equations 84 (1990), no. 2, 252-272. MR 1047569 Zbl 0778.35109

[2] G. Alessandrini and R. Gaburro, Determining conductivity with special anisotropy by boundary measurements. SIAM J. Math. Anal. 33 (2001), no. 1, 153-171. MR 1857992 Zbl 0985.35104

[3] G. Alessandrini and R. Gaburro, The local Calderón problem and the determination at the boundary of the conductivity. Comm. Partial Differential Equations 34 (2009), no. 7-9, 918-936. MR 2560305 Zbl 1196.35227

[4] K. Astala, M. Lassas, and L. Päivärinta, Calderón's inverse conductivity problem in the plane. Ann. of Math. (2) 163 (2006), no. 1, 265-299. MR 2195135 Zbl 1111.35004

[5] H. Brezis, Functional analysis, Sobolev spaces and partial differential equations. Universitext. Springer, New York, 2011. MR 2759829 Zbl 1220.46002

[6] P. Briet, H. Kovarik, G. Raikov, and E. Soccorsi, Eigenvalue asymptotics in a twisted waveguide. Comm. Partial Differential Equations 34 (2009), no. 7-9, 818-836. MR 2560302 Zbl 1195.35119

[7] M. Choulli, Y. Kian, and E. Soccorsi, Stable determination of time-dependent scalar potential from boundary measurements in a periodic quantum waveguide. arXiv:1306.6601 [math.AP]

[8] J. Dacles-Mariani and G. H. Rodrigue, Study of optically induced effects due to bending and twisting using the vector finite-element method. J. Opt. Soc. Amer. B, Opt. Phys. 23 (2006), 1743-1751.

[9] D. Dos Santos Ferreira, C. E. Kenig, M. Salo, and G. Uhlmann, Limiting Carleman weights and anisotropic inverse problems. Invent. math. 178 (2009), 119-171. MR 2534094 Zbl 1181.35327

[10] D. Dos Santos Ferreira, Y. Kurylev, M. Lassas, and M. Salo, The Calderón problem in transversally anisotropic geometries. Preprint 2013. arXiv:1305.1273 [math.AP]

[11] T. Ekholm, H. Kovarik, and D. Krejcirik, A Hardy inequality in twisted waveguides. Arch. Ration. Mech. Anal. 188 (2008), no. 2, 245-264. MR 2385742 Zbl 1167.35026

[12] R. Gaburro and W. Lionheart, Recovering Riemannian metrics in monotone families from boundary data. Inverse Problems 25 (2009), no. 4, 045004, 14 pp. MR 2482155 Zbl 1172.35078 
[13] V. Girault and P.-A. Raviart, Finite element methods for Navier-Stokes equations. Theory and algorithms. Springer Series in Computational Mathematics, 5. Springer, Berlin etc., 1986. MR 0851383 Zbl 0585.65077

[14] P. Grisvard, Elliptic problems in nonsmooth domains. Monographs and Studies in Mathematics, 24. Pitman Advanced Publishing Program. Pitman, Bost etc., 1985. MR 0775683 Zbl 0695.35060

[15] C. Guillarmou and A. Sà Barreto, Inverse problems for Einstein manifolds. Inverse Probl. Imaging 3 (2009), no. 1, 1-15. MR 2558301 Zbl 1229.58025

[16] S. Hofmann and S. Kim, The Green function estimates for strongly elliptic systems of second order. Manuscripta Math. 124 (2007), no. 2, 139-172. MR 2341783 Zbl 1130.35042

[17] L. Hörmander, The analysis of linear partial differential operators. I. Distribution theory and Fourier analysis. $2^{\text {nd }}$ ed. Grundlehren der Mathematischen Wissenschaften, 256. Springer, Berlin etc., 1990. MR 1065993 Zbl 0712.35001

[18] O. Imanuvilov, G. Uhlmann, and M. Yamamoto, Determination of second-order elliptic operators in two dimensions from partial Cauchy data. Proc. Natl. Acad. Sci. USA 108 (2011), no. 2, 467-472. MR 2770947 Zbl 1256.35203

[19] O. Imanuvilov, G. Uhlmann, and M. Yamamoto, Partial Cauchy data for general second order elliptic operators in two dimensions. Publ. Res. Inst. Math. Sci. 48 (2012), no. 4, 971-1055. MR 2999548 Zbl 1260.35253

[20] Y. W. Kang, Twisted waveguide accelerating structure. AIP Conf. Proc. 569 (2001), 335-339.

[21] H. Kovarik, D. Krejcirik, A Hardy inequality in a twisted Dirichlet-Neumann waveguide. Math. Nachr. 281 (2008), no. 8, 1159-1168. MR 2427167 Zbl 1171.35084

[22] D. Krejcirik and E. Zuazua, The Hardy inequality and the heat equation in twisted tubes. J. Math. Pures Appl. (9) 94 (2010), no. 3, 277-303. MR 2679028 Zbl 1217.35029

[23] D. Krejcirik and E. Zuazua, The asymptotic behaviour of the heat equation in a twisted Dirichlet-Neumann waveguide. J. Differential Equations 250 (2011), no. 5, 2334-2346. MR 2756066 Zbl 1213.35107

[24] Y. W. Kang and A. E. Fathy, Twisted waveguides for particle accelerator applications. Microwave Symposium Digest, MTT '09. IEEE MTT-S International, 2009, 129-132.

[25] H. Kovařík and A. Sacchetti, Resonances in twisted quantum waveguides. $J$. Phys. A 40 (2007), no. 29, 8371-8384. MR 2371239 Zbl 1117.81057

[26] M. Lassas and G. Uhlmann, On determining a Riemannian manifold from the Dirichlet-to-Neumann map. Ann. Sci. École Norm. Sup. (4) 34 (2001), no. 5, 771-787. MR 1862026 Zbl 0992.35120

[27] J. Lee and G. Uhlmann, Determining anisotropic real-analytic conductivities by boundary measurements. Comm. Pure Appl. Math. 42 (1989), no. 8, 1097-1112. MR 1029119 Zbl 0702.35036 
[28] W. R. B. Lionheart, Conformal uniqueness results in anisotropic electrical impedance imaging. Inverse Problems 13 (1997), no. 1, 125-134. MR 1435872 Zbl 0868.35140

[29] J.-L. Lions and E. Magenes, Problèmes aux limites non homogènes et applications. Vol. 1. Travaux et Recherches Mathématiques, No. 17. Dunod, Paris, 1968. MR 0247243 Zbl 0165.10801

[30] C. Miranda, Partial differential equations of elliptic type. $2^{\text {nd }}$ revised ed. Translated from the Italian by Z. C. Motteler. Ergebnisse der Mathematik und ihrer Grenzgebiete, Band 2. Springer, Berlin etc., 1970. MR 0284700 Zbl 0198.14101

[31] A. Nicoleta, F. Zolla, S. Guenneau, Modelling of twisted optical waveguides with edge elements. Eur. Phys. J. Appl. Phys. 28 (2004), no. 2, 153-157.

[32] L. Schwartz, Analyse Deuxième partie: Topologie générale et analyse fonctionnelle. Collection Enseignement des Sciences, No. 11. Hermann, Paris, 1970. MR 0467223 Zbl 0206.06301

[33] D. M. Shyroki, Exact equivalent straight waveguide model for bent and twisted waveguides. IEEE Trans. on Microwave Theory and Techniques 56 (2008), no. 2, 414-419.

[34] Z. Sun and G. Uhlmann, Anisotropic inverse problems in two dimensions. Inverse Problems 19 (2003), no. 5, 1001-1010. MR 2024685 Zbl 1054.35139

[35] J. Sylvester, An anisotropic inverse boundary value problem. Comm. Pure Appl. Math. 43 (1990), no. 2, 201-232. MR 1038142 Zbl 0709.35102

[36] J. Sylvester and G. Uhlmann, A global uniqueness theorem for an inverse boundary value problem. Ann. of Math. (2) 125 (1987), no. 1, 153-169. MR 0873380 Zbl 0625.35078

[37] J. L. Wilson, Analysis of rapidly twisted hollow waveguides. IEEE Trans. on Microwave Theory and Techniques 57 (2009), no. 1, 130-139.

[38] H. Yabe and Y. Mushiake, An analysis of a hybrid-mode in a twisted rectangular waveguide. IEEE Trans. on Microwave Theory and Techniques 32 (1984), no. 1, 65-71.

Received September 3, 2013; revised July 23, 2014

Mourad Choulli, Institut Élie Cartan de Lorraine, UMR CNRS 7502, Université de Lorraine, Ile du Saulcy, F-57045 Metz cedex 1, France e-mail: mourad.choulli@univ-lorraine.fr

Eric Soccorsi, Aix-Marseille Université, CNRS, CPT UMR 7332, 13288 Marseille, France \& Université de Toulon, CNRS, CPT UMR 7332, 83957 La Garde, France e-mail: eric.soccorsi@univ-amu.fr 